\title{
Imprint of the symmetry energy on the inner crust and strangeness content of neutron stars
}

\author{
Constança Providência ${ }^{1}$, Sidney S. Avancini ${ }^{2}$, Rafael Cavagnoli ${ }^{3}$, Silvia Chiacchiera ${ }^{1}$, Camille Ducoin ${ }^{4}$, Fabrizio \\ Grill $^{1}$, Jérôme Margueron ${ }^{4}$, Débora P. Menezes ${ }^{2}$, Aziz Rabhi $^{1,5}$, and Isaac Vidaña ${ }^{1}$ \\ 1 Centro de Física Computacional, Department of Physics, University of Coimbra, P3004-516 Coimbra, Portugal, \\ 2 Departamento de Física, CFM, Universidade Federal de Santa Catarina, Florianópolis - SC - CP. 476, CEP 88.040 - 900, \\ Brazil, \\ 3 Departamento de Física, IFM, Universidade Federal de Pelotas, Pelotas/SC, CP 354, CEP 96001-970, Brazil \\ 4 Institut de Physique Nucléaire de Lyon, CNRS/IN2P3, Université Claude Bernard Lyon 1, F-69622 Villeurbanne, France \\ ${ }^{5}$ Laboratoire de Physique de la Matière Condensée, Faculté des Sciences de Tunis, Campus Universitaire, Le Belvédère-1060, \\ Tunisia,
}

Received: date / Revised version: date

\begin{abstract}
In this work we study the effect of the symmetry energy on several properties of neutron stars. First, we discuss its effect on the density, proton fraction and pressure of the neutron star crust-core transition. We show that whereas the first two quantities present a clear correlation with the slope parameter $L$ of the symmetry energy, no satisfactory correlation is seen between the transition pressure and $L$. However, a linear combination of the slope and curvature parameters at $\rho=0.1 \mathrm{fm}^{-3}$ is well correlated with the transition pressure. In the second part we analyze the effect of the symmetry energy on the pasta phase. It is shown that the size of the pasta clusters, number of nucleons and the cluster proton fraction depend on the density dependence of the symmetry energy: a small $L$ gives rise to larger clusters. The influence of the equation of state at subsaturation densities on the extension of the inner crust of the neutron star is also discussed. Finally, the effect of the effect of the density dependence of the symmetry energy on the strangeness content of neutron stars is studied in the last part of the work. It is found that charged (neutral) hyperons appear at smaller (larger) densities for smaller values of the slope parameter $L$. A linear correlation between the radius and the strangeness content of a star with a fixed mass is also found.
\end{abstract}

PACS. 26.60.-c Nuclear matter aspects of neutron stars - 21.65.-f Nuclear matter

\section{Introduction}

Isospin asymmetric nuclear matter is present in nuclei, especially in those far away from the stability line, and in astrophysical systems, particularly in neutron stars. Therefore, a well-grounded understanding of the properties of isospin-rich nuclear matter is a necessary ingredient for the advancement of both nuclear physics and astrophysics [1, 2, 3. Nevertheless, in spite of the experimental [4] and theoretical [5] efforts carried out to study the properties of isospin-asymmetric nuclear systems, some of these properties are not well constrained yet. In particular, the density dependence of the symmetry energy is still an important source of uncertainties. In this work, we study how the density dependence of the symmetry energy affects the equation of state (EOS) of asymmetric nuclear matter. We will focus on three different problems.

In the first part the correlations of the slope and curvature parameters of the symmetry energy with the density, proton fraction and pressure at the neutron star crust-core transition are analyzed. The analysis is done with the mi- croscopic Brueckner-Hartree-Fock (BHF) approach and several phenomenological Skyrme and relativistic mean field (RMF) models to describe the nuclear EOS [6]. A generalized liquid drop model (GLDM) based on a density development around a reference density is introduced to allow the identification of possible existing correlations between the crust-core transition and a limited set of the coefficients of this development 7,8 . We show that the transition density and the transition proton fraction are correlated with the symmetry energy slope parameter $L$ and that the transition pressure shows no clear correlation with the symmetry energy slope at saturation. Nevertheless, a correlation exists between the transition pressure and a linear combination of the symmetry energy slope and curvature defined at $\rho=0.1 \mathrm{fm}^{-3}$.

In the second part the effect of the density dependence of the symmetry energy on the pasta phase is discussed. It is shown that the number of nucleons in the clusters, the cluster proton fraction and the size of the Wigner Seitz cell are very sensitive to the density dependence of the symmetry energy, and that the symmetry energy slope 
parameter $L$ may have quite dramatic effects on the cell structure if it is very large or small [9, 10, 11]. Rod-like and slab-like pasta clusters have been obtained in all models except one, with a large slope parameter $L$. The effect of $L$ on the extension of the inner crust is also discussed. In particular, it is shown that a smaller $L$ favors a wider slab phase. This phase may allow the propagation of low frequency modes that will affect the specific heat in a nonnegligible way [12.

In the last part, the effect of the density dependence of the symmetry energy on the strangeness content of a neutron star is studied. The study is done with RMF models. It is shown that there is still lacking information on the nucleonic equation of state at supra-saturation densities and, in particular, on the hyperon interactions in nuclear matter. Therefore, the role of exotic degrees of freedom on the interior of compact stars is still an open issue [13, 14, 15. 16 .

Finally, we show that some star properties are affected in a similar way by the density dependence of the symmetry energy and the hyperon content of the star. A linear correlation between the radius and the strangeness content of a star with a fixed mass is obtained [16.

In the following, before considering each one of these issues, we briefly review the main features of the asymmetric nuclear matter (ANM) equation of state and the different models considered in this work.

\section{Asymmetric Nuclear Matter EOS}

Assuming charge symmetry of the nuclear forces, the energy per particle of ANM can be expanded on the isospin asymmetry parameter, $\delta=(N-Z) /(N+Z)=\left(\rho_{n}-\rho_{p}\right) / \rho$, around the values of symmetric nuclear matter $(\delta=0)$ in terms of even powers of $\delta$ as

$$
E(\rho, \delta) \simeq E_{S N M}(\rho)+E_{\text {sym }}(\rho) \delta^{2}
$$

where $E_{S N M}(\rho)$ is the energy per particle of symmetric matter and $E_{\text {sym }}(\rho)=\left.\frac{1}{2} \frac{\partial^{2} E}{\partial \delta^{2}}\right|_{\delta=0}$ is the symmetry energy.

It is common to characterize the density dependence of the symmetry energy around the saturation density $\rho_{0}$ in terms of a few bulk parameters by expanding it in a Taylor series

$$
E_{\text {sym }}(\rho)=J+L\left(\frac{\rho-\rho_{0}}{3 \rho_{0}}\right)+\frac{K_{\text {sym }}}{2}\left(\frac{\rho-\rho_{0}}{3 \rho_{0}}\right)^{2}+\mathcal{O}(3),
$$

where $J$ is the value of the symmetry energy at saturation and the quantities $L$ and $K_{\text {sym }}$ are related to its slope and curvature, respectively, at such density,

$$
L=\left.3 \rho_{0} \frac{\partial E_{\text {sym }}(\rho)}{\partial \rho}\right|_{\rho=\rho_{0}}, K_{\text {sym }}=\left.9 \rho_{0}^{2} \frac{\partial^{2} E_{\text {sym }}(\rho)}{\partial \rho^{2}}\right|_{\rho=\rho_{0}} .
$$

\subsection{The BHF approach of ANM}

The BHF approach of ANM [17] starts with the construction of all the $G$ matrices which describe the effective interaction between two nucleons in the presence of a surrounding medium. They are obtained by solving the well known Bethe-Goldstone equation, schematically written as

$$
\begin{aligned}
G_{\tau_{1} \tau_{2} ; \tau_{3} \tau_{4}}(\omega) & =V_{\tau_{1} \tau_{2} ; \tau_{3} \tau_{4}}+\sum_{i j} V_{\tau_{1} \tau_{2} ; \tau_{i} \tau_{j}} \\
& \times \frac{Q_{\tau_{i} \tau_{j}}}{\omega-\epsilon_{i}-\epsilon_{j}+i \eta} G_{\tau_{i} \tau_{j} ; \tau_{3} \tau_{4}}(\omega)
\end{aligned}
$$

where $\tau=n, p$ indicates the isospin projection of the two nucleons in the initial, intermediate and final states, $V$ denotes the bare NN interaction, $Q_{\tau_{i} \tau_{j}}$ the Pauli operator that allows only intermediate states compatible with the Pauli principle, and $\omega$, the so-called starting energy, corresponds to the sum of non-relativistic energies of the interacting nucleons. The single-particle energy $\epsilon_{\tau}$ of a nucleon with momentum $\boldsymbol{k}$ is given by

$$
\epsilon_{\tau}(\boldsymbol{k})=\frac{\hbar^{2} k^{2}}{2 m_{\tau}}+\operatorname{Re}\left[U_{\tau}(\boldsymbol{k})\right]
$$

where the single-particle potential $U_{\tau}(\boldsymbol{k})$ represents the mean field "felt" by a nucleon due to its interaction with the other nucleons of the medium. In the BHF approximation, $U(\boldsymbol{k})$ is calculated through the "on-shell energy" $G$-matrix, and is given by

$$
\begin{aligned}
U_{\tau}(\boldsymbol{k})= & \sum_{\tau^{\prime}} \sum_{\left|\boldsymbol{k}^{\prime}\right|<k_{F_{\tau^{\prime}}}} \\
& \left\langle\boldsymbol{k} \boldsymbol{k}^{\prime}\left|G_{\tau \tau^{\prime} ; \tau \tau^{\prime}}\left(\omega=\epsilon_{\tau}(k)+\epsilon_{\tau^{\prime}}\left(k^{\prime}\right)\right)\right| \boldsymbol{k} \boldsymbol{k}^{\prime}\right\rangle_{A}
\end{aligned}
$$

where the sum runs over all neutron and proton occupied states and where the matrix elements are properly antisymmetrized. We note here that the so-called continuous prescription has been adopted for the single-particle potential when solving the Bethe-Goldstone equation [18]. Once a self-consistent solution of Eqs. (4)-(6) is achieved, the energy per particle can be calculated as

$$
E(\rho, \delta)=\frac{1}{A} \sum_{\tau} \sum_{|\boldsymbol{k}|<k_{F_{\tau}}}\left(\frac{\hbar^{2} k^{2}}{2 m_{\tau}}+\frac{1}{2} \operatorname{Re}\left[U_{\tau}(\boldsymbol{k})\right]\right) .
$$

The BHF calculation carried out in this work uses the realistic Argonne V18 [19] nucleon-nucleon interaction supplemented with a nucleon three-body force of Urbana type which, for the use in BHF calculations, was reduced to a two-body density dependent force by averaging over the spatial, spin and isospin coordinates of the third nucleon in the medium [20].

\subsection{Phenomenological models}

Phenomenological approaches, either relativistic or nonrelativistic, are based on effective interactions that are frequently built to reproduce properties of nuclei. Skyrme interactions 21] and RMF models 22] are among the most 
commonly used ones. They are briefly described in the next.

\subsubsection{Skyrme interaction}

The standard form of a Skyrme interaction reads

$$
\begin{aligned}
V\left(\mathbf{r}_{1}, \mathbf{r}_{2}\right) & =t_{0}\left(1+x_{0} P^{\sigma}\right) \delta(\mathbf{r}) \\
& +\frac{1}{2} t_{1}\left(1+x_{1} P^{\sigma}\right)\left(\mathbf{k}^{\prime 2} \delta(\mathbf{r})+\delta(\mathbf{r}) \mathbf{k}^{2}\right) \\
& +t_{2}\left(1+x_{2} P^{\sigma}\right) \mathbf{k}^{\prime} \cdot \delta(\mathbf{r}) \mathbf{k} \\
& +\frac{1}{6} t_{3}\left(1+x_{3} P^{\sigma}\right)(\rho(\mathbf{R}))^{\alpha} \delta(\mathbf{r}) \\
& +i W_{0}\left(\sigma_{1}+\sigma_{2}\right)\left(\mathbf{k}^{\prime} \times \delta(\mathbf{r}) \mathbf{k}\right)
\end{aligned}
$$

where $\mathbf{r}=\mathbf{r}_{1}-\mathbf{r}_{1}, \mathbf{R}=\left(\mathbf{r}_{1}+\mathbf{r}_{\mathbf{2}}\right) / \mathbf{2}, \mathbf{k}=\left(\nabla_{1}-\nabla_{2}\right) / 2 i$ is the relative momentum acting on the right, $\mathbf{k}^{\prime}$ its conjugate acting on the left and $P^{\sigma}=\left(1+\sigma_{\mathbf{1}} \cdot \sigma_{\mathbf{2}}\right) / \mathbf{2}$ is the spin exchange operator. The last term, proportional to $W_{0}$, corresponds to the zero-range spin-orbit term. It does not contribute in homogeneous systems and thus will be ignored for the rest of this article.

Most of these forces are, by construction, well behaved close to the saturation density and moderate isospin asymmetries. Nevertheless, only certain combinations of their parameters are well determined empirically. Consequently, there is a proliferation of different Skyrme interactions that produce a similar EOS for symmetric nuclear matter but predict a very different one for pure neutron matter. A few years ago, Stone et al., 23 tested extensively and systematically the capabilities of almost 90 existing Skyrme parametrizations to provide good neutron star candidates. They found that only 27 of these parametrizations passed the restricted tests they imposed, the key property being the density dependence of the symmetry energy. These forces are SLy0-SLy10 24] and SLy230a 25] of the Lyon group, SkI1-SkI5 [26] and SkI6 [27] of the SkI family, Rs and Gs 28, SGI [29], SkMP [30], SkO and SkO' 31, SkT4 and SkT5 [32], and the early SV [33]. The results for the Skyrme forces shown in this work have been obtained with these 27 forces and the additional parametrizations SGII 29], RATP 34, SLy230b 25], NRAPR [3], LNS 35, BSk14 36, BSk16 37] and BSk17 38. We should mention, however, that more stringent constraints to the Skyrme forces have been very recently presented by Dutra et al., in Ref. 39. These authors have examined the suitability of 240 Skyrme interactions with respect to eleven macroscopic constraints derived mainly from experimental data and the empirical properties of symmetric nuclear matter at and close to saturation. They have found that only 5 of the 240 forces analyzed satisfy all the constraints imposed. We note that among the parametrizations used in this work, only the NRAPR and LNS ones belong to this restricted set.

\subsubsection{RMF models}

RMF models are based on effective Lagrangian densities where nucleons interact with and through an isoscalar- scalar field $\sigma$, an isoscalar-vector field $\omega^{\mu}$, an isovectorvector field $\boldsymbol{\rho}^{\mu}$, and an isovector-scalar field $\boldsymbol{\delta}$. In this work we consider models with constant couplings and non-linear terms 40, and with density dependent couplings 41. Within the first class of models, that we will designate by Non Linear Walecka Models (NLWM), we consider NL3 42 and GM1, GM3 45] with non linear $\sigma$ terms, NL3 ${ }_{\omega \rho}$ including also non-linear $\omega \rho$ terms that allow the modulation of the density dependence of the symmetry energy 44, TM1 43, with non linear $\sigma$ and $\omega$ terms, FSU [46] and IU-FSU [4] with non-linear $\sigma, \omega$ and $\omega \rho$ terms. The last two parametrizations were constrained by the collective response of nuclei to the isoscalar monopole giant resonance (ISGMR) and the isovector dipole giant resonance (IVGDR). Within the second class of models with density dependent couplings we consider TW 41, DD-ME2 48 and DD-ME $\delta$ 49]: DD-ME2, as all the nonlinear parametrizations considered, does not include the $\delta$ meson, and was adjusted to experimental data based on finite nuclei properties; DD-ME $\delta$ contains the $\delta$ meson and was fitted to microscopic ab-initio calculations in nuclear matter and finite nuclei properties. Both models present similar properties for the symmetry energy, however, DDME2 has a larger incompressibility at saturation.

The Lagrangian density for these models typically reads

$$
\mathcal{L}=\sum_{i=p, n} \mathcal{L}_{i}+\mathcal{L}_{\sigma}+\mathcal{L}_{\omega}+\mathcal{L}_{\rho}+\mathcal{L}_{\delta}+\mathcal{L}_{n l}
$$

where the nucleon Lagrangian is

$$
\mathcal{L}_{i}=\bar{\psi}_{i}\left[\gamma_{\mu} i D^{\mu}-M^{*}\right] \psi_{i}
$$

with

$$
\begin{aligned}
i D^{\mu} & =i \partial^{\mu}-\Gamma_{\omega} \Omega^{\mu}-\frac{\Gamma_{\rho}}{2} \boldsymbol{\tau} \cdot \boldsymbol{\rho}^{\mu}, \\
M^{*} & =M-\Gamma_{\sigma} \sigma-\Gamma_{\delta} \boldsymbol{\tau} \cdot \boldsymbol{\delta},
\end{aligned}
$$

and the meson Lagrangian densities are given by

$$
\begin{aligned}
\mathcal{L}_{\sigma} & =\frac{1}{2}\left(\partial_{\mu} \sigma \partial^{\mu} \sigma-m_{\sigma}^{2} \sigma^{2}\right) \\
\mathcal{L}_{\omega} & =\frac{1}{2}\left(-\frac{1}{2} \Omega_{\mu \nu} \Omega^{\mu \nu}+m_{\omega}^{2} \omega_{\mu} \omega^{\mu}\right) \\
\mathcal{L}_{\rho} & =\frac{1}{2}\left(-\frac{1}{2} \mathbf{R}_{\mu \nu} \cdot \mathbf{R}^{\mu \nu}+m_{\rho}^{2} \boldsymbol{\rho}_{\mu} \cdot \boldsymbol{\rho}^{\mu}\right) \\
\mathcal{L}_{\delta} & =\frac{1}{2}\left(\partial_{\mu} \boldsymbol{\delta} \partial^{\mu} \boldsymbol{\delta}-m_{\delta}^{2} \boldsymbol{\delta}^{2}\right) \\
\mathcal{L}_{n l} & =-\frac{1}{3 !} \kappa \sigma^{3}-\frac{1}{4 !} \lambda \sigma^{4}+\frac{1}{4 !} \xi \Gamma_{\omega}^{4}\left(\omega_{\mu} \omega^{\mu}\right)^{2} \\
& +\Lambda_{\omega} \Gamma_{\omega}^{2} \Gamma_{\rho}^{2} \omega_{\mu} \omega^{\mu} \boldsymbol{\rho}_{\mu} \cdot \boldsymbol{\rho}^{\mu} .
\end{aligned}
$$

In the above equations $\Gamma_{i}(i=\sigma, \omega, \rho, \delta)$ denote, depending on the model, the constant or density-dependent coupling parameters. Finally, we note that the photon and electron Lagrangian densities

$$
\begin{aligned}
\mathcal{L}_{\gamma} & =-\frac{1}{4} F_{\mu \nu} F^{\mu \nu} \\
\mathcal{L}_{e} & =\bar{\psi}_{e}\left[\gamma_{\mu}\left(i \partial^{\mu}+e A^{\mu}\right)-m_{e}\right] \psi_{e}
\end{aligned}
$$


and the term $-e\left(1+\tau_{3}\right) A^{\mu} / 2$ should be added to Eqs. (9) and (11), respectively, when describing non-homogeneous matter. $\beta$-equilibrium matter requires the inclusion of the lepton Lagrangian density only.

\subsection{The Generalized Liquid-Drop Model (GLDM)}

The liquid-drop model equation of state is based on a density expansion around the saturation point, its main features being: the saturation density $\rho_{0}$, the energy per nucleon at saturation $E_{0}$, the incompressibility coefficient $K_{0}$ which characterizes the curvature of the EOS, and the isovector coefficients, namely the symmetry energy $J$, its slope $L$ and the symmetry incompressibility $K_{\text {sym }}$. We focus on the bulk-matter equation of state, and no surface effects are considered. In order to discuss the link that can be drawn between laboratory constraints and EOS properties in situations quite away from the experimental data, in particular, at high density or at low proton fraction, we introduce a "generalized liquid-drop model" (GLDM) 7, 8, still addressing the bulk EOS, which expresses the EOS as an expansion of arbitrary order around a chosen reference density $\rho_{\text {ref }}$ (not necessarily the saturation density $\left.\rho_{0}\right)$ :

$$
\begin{aligned}
E_{\mathrm{GLDM}}(\rho, \delta) & =\sum_{n=0}^{\mathcal{N}}\left(c_{\mathrm{IS}, n}+c_{\mathrm{IV}, n} \delta^{2}\right) \frac{x^{n}}{n !} \\
& +\left(E_{\mathrm{kin}}-E_{\mathrm{kin}}^{\mathrm{para}}\right),
\end{aligned}
$$

with $x=\left(\rho-\rho_{\text {ref }}\right) / 3 \rho_{\text {ref }}$. The coefficients $c_{\mathrm{IS}, n}$ and $c_{\mathrm{IV}, n}$, where the index 'IS' ('IV') stands for isoscalar (isovector), are associated with the derivatives of the energy $E(\rho, \delta=$ $0)$ and of the symmetry energy $E_{\text {sym }}(\rho)$

$$
\begin{aligned}
& c_{\mathrm{IS}, n}\left(\rho_{\text {ref }}\right)=\left(3 \rho_{\text {ref }}\right)^{n} \frac{\partial^{n} E}{\partial \rho^{n}}\left(\rho_{\text {ref }}, 0\right) \\
& c_{\text {IV }, n}\left(\rho_{\text {ref }}\right)=\left(3 \rho_{\text {ref }}\right)^{n} \frac{\partial^{n} E_{\text {sym }}}{\partial \rho^{n}}\left(\rho_{\text {ref }}\right) .
\end{aligned}
$$

For $\rho_{\text {ref }}=\rho_{0}$, the lower-order coefficients are usual nuclear matter properties: $c_{\mathrm{IS}, 0}=E_{0}$ (saturation energy), $c_{\mathrm{IS}, 2}=$ $K_{0}$ (incompressibility), $c_{\mathrm{IS}, 3}=Q_{0}$ (skewness), $c_{\mathrm{IV}, 0}=J$ (symmetry energy), $c_{\mathrm{IV}, 1}=L$ (symmetry-energy slope), $c_{\mathrm{IV}, 2}=K_{\mathrm{sym}}$ (symmetry incompressibility), $c_{\mathrm{IV}, 3}=Q_{\mathrm{sym}}$. Since the effective laboratory constraints are rather related to subsaturation density, the coefficients of a GLDM with reference density $\rho_{\text {ref }}<\rho_{0}$ are expected to be better constrained than the standard saturation coefficients. Also, the neutron-star core-crust transition properties are better correlated with coefficients defined at subsaturation density because the reference point is closer to the transition point.

The isovector channel of the EOS in Eq. (20) has a parabolic contribution accounted for by the isovector coefficients multiplied by $\delta^{2}$, and a minimal extra-parabolic correction, the model-independent kinetic term $E_{\text {kin }}-E_{\text {kin }}^{\text {para }}$,

$$
E_{\mathrm{kin}}=\frac{\left(3 \pi^{2} / 2\right)^{5 / 3}}{10 m \pi^{2}} \rho^{2 / 3}\left[(1+\delta)^{5 / 3}+(1-\delta)^{5 / 3}\right]
$$

$$
E_{\mathrm{kin}}^{\mathrm{para}}=\frac{\left(3 \pi^{2} / 2\right)^{5 / 3}}{10 m \pi^{2}} \rho^{2 / 3}\left[2+\frac{10}{9} \delta^{2}\right]
$$

that introduces the divergence of energy-density curvature in the proton-density direction at small proton density and, therefore, avoids that the spinodal contour reaches pure neutron matter. In the above equation $m$ refers to the nucleon mass.

The EOS obtained with any nuclear model can be associated with its corresponding GLDM. In the limit of an infinite expansion $\left(\mathrm{GLDM}_{\infty}\right)$, the symmetric matter EOS $E(\rho, 0)$ and the symmetry energy $E_{\text {sym }}(\rho)$ are exactly equivalent to the complete model EOS, the only remaining difference, for the EOS of asymmetric matter, being the extra-parabolic terms of the interaction part. In the following, we will address the correlations that could be found between GLDM coefficients and the neutron-star core-crust transition properties, being focused, in particular, on the isovector coefficients.

\section{Crust-core transition}

The EOS of nuclear matter can be constrained by laboratory data and astrophysical observations. It has been shown in Refs. 7] and 8 that an accurate determination of the symmetry energy and its slope and curvature at a subsaturation density, $\rho=0.1 \mathrm{fm}^{-3}$, allows a quite accurate prediction of the core-crust transition properties.

Nuclear models could then be used to restrict the range of the core-crust transition properties in neutron stars and contribute to the interpretation of astrophysical observations. Pulsar glitches are an example 50, since the transition pressure is an essential input to infer the neutron-star mass-radius relation from glitch observations.

In the present section we will show how some of the EOS properties are correlated with the crust-core transition properties.

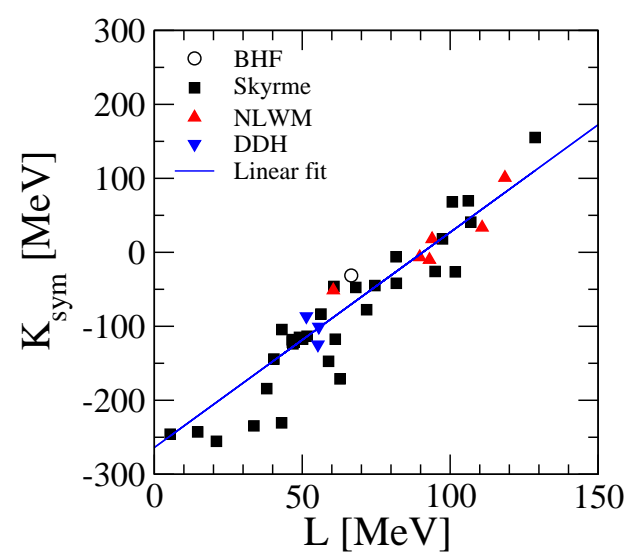

Fig. 1. (Color online) Correlation between $K_{\text {sym }}$ and $L$. 


\subsection{Correlation between $L$ and $K_{\mathrm{sym}}$}

When different nuclear models are compared, the GLDM coefficients taken at saturation density present some correlations between them. This is a manifestation of the fact that the effective constraints from laboratory data do not fix the nuclear properties at saturation, but rather at a lower density. Indeed, the nuclei whose properties are used to fit the effective nuclear density functionals have a mean density that is lower than $\rho_{0}$. Many different nuclear models tend to converge to a symmetry energy close to $25 \mathrm{MeV}$ at $\rho \simeq 0.12 \mathrm{fm}^{-3}$. A similar convergence can be observed for the symmetry energy slope, whose value becomes close to $100 \mathrm{MeV}$ at $\rho \simeq 0.06 \mathrm{fm}^{-3}$ (see Refs. 7] and [8] for a more detailed analysis of these features).

These convergent trends away from the saturation point imply some correlations in the expansion coefficients around saturation density. An example is the existing correlation between $J$ and $L$ [7, 8 : since the symmetry energy is better constrained at subsaturation density, higher values of $L$ have to be compensated by higher values of $J$. Another example is the correlation between $L$ and $K_{\text {sym }}$ shown in Fig. 1. The remarkably strong $L-K_{\text {sym }}$ correlation plays an important role in the links that can be drawn between the GLDM coefficients (namely, laboratory constraints) and the core-crust transition pressure in neutron star, a sensitive input for the interpretation of pulsar glitches.

\subsection{Correlation between $L$ and the core-crust transition properties}

New experimental perspectives for the measurement of $L$ have drawn interest in trying to correlate this quantity to the core-crust transition properties. Such correlations have to be considered with care, taking into account that fake relations may appear when the study is limited to a restricted nuclear model or family of models, with internal correlations that disappear if different kinds of functional models are considered. Reliable correlations between GLDM coefficients and the core-crust transition properties have to remain true independently of the difference between nuclear models, as long as these models account for the existing experimental constraints.

Studying the correlations between $L$ and the core-crust transition properties in the framework of various models (effective relativistic and Skyrme models, and BHF calculations), we arrive to the conclusion that: (i) $L$ is well correlated with the transition density and proton fraction $\left(\rho_{\mathrm{t}}, Y_{\mathrm{p}, \mathrm{t}}\right)$ and (ii) $L$ is not satisfactorily correlated with the transition pressure $P_{\mathrm{t}}$. We have denoted the proton fraction by $Y_{p}=\rho_{p} / \rho$, and used the subscript $t$ to refer to the properties at the crust-core transition. We summarize below the explanation of this situation (see Refs. [7,8] for a more detailed analysis).

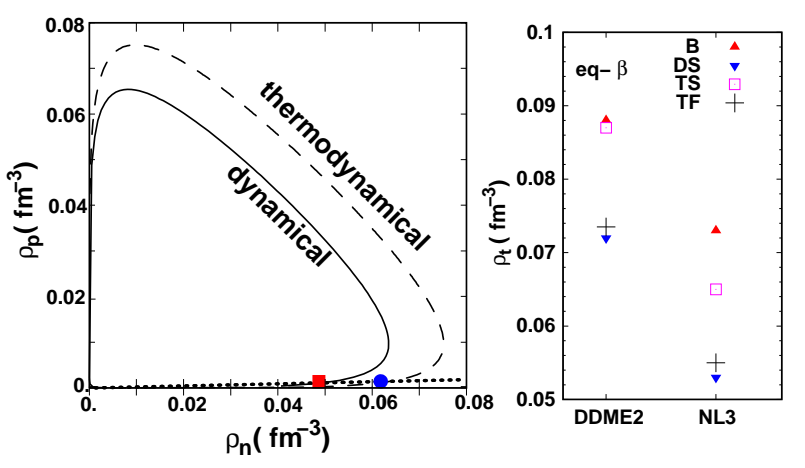

Fig. 2. (Color online) Left panel: Comparison between the thermodynamical (dashed line) and dynamical (full line) spinodals. The dotted line represents the $\beta$-equilibrium EOS and the red square and blue dot define the crust-core transition within, respectively, the dynamical and thermodynamical spinodal. Right panel: comparison of the transition density obtained from different approaches (binodal, dynamical spinodal, thermodynamical spinodal and Thomas Fermi calculation) for two RMF models: DDME2 and NL3.

\subsubsection{Core-crust transition: thermodynamical versus dynamical calculations}

We will next discuss how to determine the core-crust transition point in neutron stars. Cold neutron-star matter is in $\beta$-equilibrium, and is transparent to neutrinos, thus, for a given nuclear density, the proton fraction of homogeneous nuclear matter is essentially determined by the symmetry energy at this density. The core-crust border is the transition from the homogeneous matter of the core to the clusterized matter of the inner crust. For the very neutron-rich matter of a cold neutron star, this transition is well approximated by the dynamical spinodal border [51, as shown in Refs. 9, 11] where it was compared with pasta phase calculations. The dynamical spinodal is the density region where the homogeneous nuclear matter is unstable against finite-size density fluctuations, eventually leading to cluster formation, and takes into account both finite size effects and the Coulomb interaction.

In fact, it is expected that the transition density lies in the metastable region between the binodal surface and the dynamical spinodal surface. The binodal surface is defined in the $\rho, Y_{p}, T$ phase space as the surface where the gas and liquid phases coexist, and corresponds to an upper limit for the extension of the pasta phase because it does not take into account neither Coulomb nor finite size effects.

A simplified definition of the transition point is determined by the crossing between the $\beta$-equilibrium line and the thermodynamical spinodal border [52,53, 54,55, 56, 57. The thermodynamical spinodal, the bulk liquidgas instability region in nuclear matter, touches the binodal surface at the critical point, which, for a given temperature, occurs at a density and proton fraction close to the crust-core transition density and proton fraction. This transition point is sensitively different from the real transition point, since the thermodynamical spinodal region is 

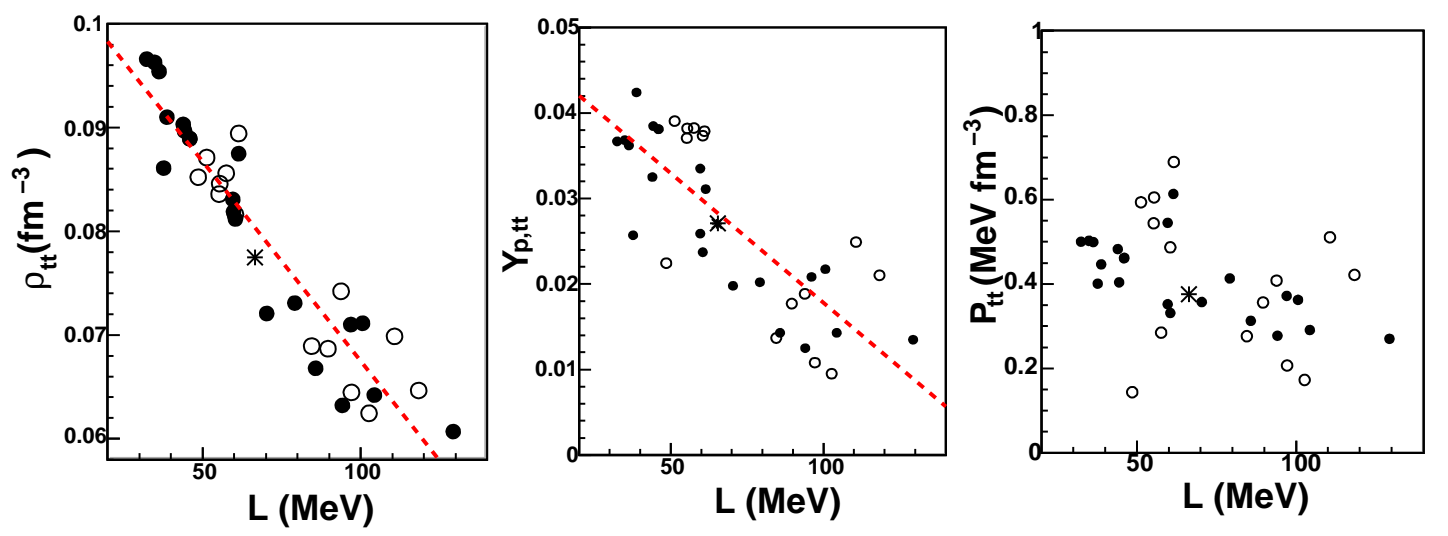

Fig. 3. (Color online) $L$ versus a) the total transition density $\rho_{t t}$ (left panel), b) the proton fraction at the transition $Y_{p, t t}$ (middle panel), and c) transition pressure $P_{t t}$ (right panel) for different nuclear models: Skyrme (full symbols), relativistic models (empty symbols), BHF (asterisk).

larger than the dynamical one, as can be seen in Fig. 2 The bulk (thermodynamical) instability of nuclear matter is at the origin of the instability against clusterization that affects star matter. This second instability region, named dynamical spinodal, takes into account surface terms and the Coulomb interaction [58, both leading to a reduction of the dynamical spinodal with respect to the bulk one. As a result, the transition point calculated on the basis of the thermodynamical spinodal (that will be denoted by the index $t t$ ) is at a significantly higher density than the actual transition point, well approximated on the basis of the dynamical spinodal (this transition point will be denoted by the index $t d$ ). However, we have verified that the properties of both transition points are well correlated [8]. Since the $t t$ point allows to study more directly the link between the GLDM coefficients and the transition properties, we start by discussing this thermodynamical transition point, keeping in mind that it represents a shifted version of the core-crust transition. The correlation effects observed in the case of the $t t$ transition are expected to apply as well to the more realistic $t d$ transition point.

\subsubsection{Correlations between $L$ and the transition density $\left(\rho_{t}, Y_{p, t}\right)$}

The correlation between $L$ and the transition density point (total density and proton fraction) is quite robust, as can be seen in Fig. 3 (left and middle panels). This results from two effects that reinforce each-other: a) a larger value of $L$ means a smaller symmetry energy at subsaturation density, i.e. a more neutron-rich $\beta$-equilibrium (lower proton fraction $\left.Y_{p, t}\right)$. According to the shape of the spinodal, this also means a lower density $\rho_{t}$; b) the value of $L$ also has an impact on the spinodal border: a larger $L$ is associated with a spinodal border at lower density. To explain this second effect, let us consider the energy-density curvature of neutron matter, taken at the symmetric matter spinodal density $\rho_{\mathrm{s}}$. This particular density is chosen in order to cancel the isoscalar terms, and to concentrate on the isovector ones. Thus, this quantity reads:

$$
\begin{aligned}
C_{\mathrm{NM}, \mathrm{s}} & =\frac{2}{3 \rho_{0}} L+\frac{1}{3 \rho_{0}} \sum_{n \geq 2} c_{\mathrm{IV}, n} \frac{x_{\mathrm{s}}^{n-2}}{(n-2) !}\left[\frac{n+1}{n-1} x_{\mathrm{s}}+\frac{1}{3}\right] \\
& +\frac{\partial^{2}\left[\rho\left(E_{\mathrm{kin}}-E_{\mathrm{kin}}^{\mathrm{para}}\right)\right]}{\partial \rho^{2}}
\end{aligned}
$$

with $x_{\mathrm{S}}=\left(\rho_{\mathrm{S}}-\rho_{0}\right) /\left(3 \rho_{0}\right)$. The leading term is proportional to $L$, and the following only has a quite weak effect (see Ref. [8]).

\subsubsection{Lack of correlation between $L$ and the transition} pressure $P_{t}$

In the case of the transition pressure $P_{t}$, no satisfactory correlation with $L$ emerges when different kinds of models are involved, as can be seen on Fig. 3 (right panel). To understand this result, it is useful to express $P$ as a development in terms of the GLDM coefficients :

$$
\begin{aligned}
P(\rho, \delta) & =\frac{\rho^{2}}{3 \rho_{0}}\left[L \delta^{2}+\sum_{n \geq 2}\left(c_{\mathrm{IS}, n}+c_{\mathrm{IV}, n} \delta^{2}\right) \frac{x^{n-1}}{(n-1) !}\right] \\
& +\rho^{2} \frac{\partial\left(E_{\mathrm{kin}}-E_{\mathrm{kin}}^{\mathrm{para}}\right)}{\partial \rho} .
\end{aligned}
$$

The lack of $L-P_{t}$ correlation that is observed results from three main effects, which are opposed and compensate each-other: a) the leading term of the density development of the pressure is proportional to $L$, so $P_{t}$ should increase with $L$; b) the transition density $\rho_{t}$ has been shown to decrease with $L$, and the pressure should decrease if the density decreases; c) the second term of the development, whose sign is negative, is proportional to the symmetry incompressibility $K_{\text {sym }}$, which is larger for larger $L$. Effects b) and c) are opposite to a).

This conclusion has been reached by analyzing the different contributions to the link between $L$ and $P_{\mathrm{t}}$ through 


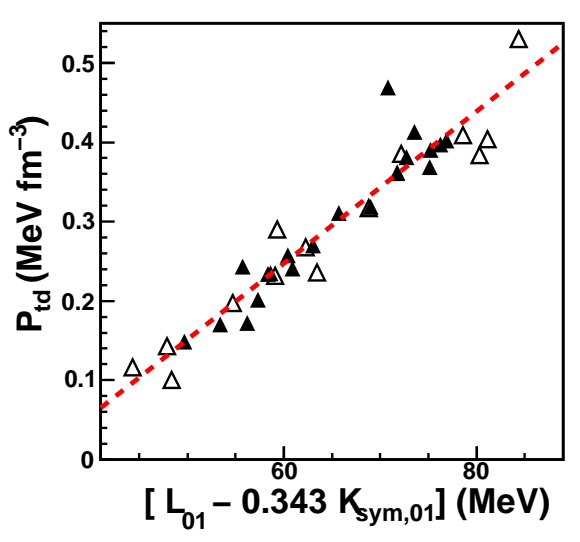

Fig. 4. (Color online) Correlation obtained between $P_{t d}$ and a linear combination of $L_{0.1}$ and $K_{\mathrm{sym}, 0.1}$ [see text, Eq. [24)].

a variational study of Eq. (22). Two kinds of variations were considered: (i) variations of the transition point (density and proton fraction) and (ii) variations of the GLDM coefficients; both kinds of variations are correlated with $L$.

Thus, for a given $L$ value, the transition pressure obtained will essentially depend on the nuclear model that is used. Different models may predict either an increasing or decreasing correlation of $P_{\mathrm{t}}$ with $L[8$, and indeed opposite predictions exist in the literature [55, 59].

\subsection{Correlations between transition pressure and GLDM coefficient combinations}

Although the link between the transition pressure and the GLDM coefficients is quite delicate, as it has been shown in the previous section, it is important to find a way to get a reliable estimation of $P_{t}$ in relation with quantities that could be constrained by laboratory data. For this reason, we looked beyond a simple correlation with $L$, which we did not obtain, and investigated the role of other GLDM coefficients (see Ref. 8] for a detailed discussion). One of the most promising correlations involves a linear combination of $L_{0.1}$ and $K_{\text {sym,0.1 }}$, denoting, respectively, the symmetry energy slope and curvature taken at the reference density $\rho_{\text {ref }}=0.1 \mathrm{fm}^{-3}$ instead of $\rho_{0}$. We have considered the pressure at the crossing of the dynamical spinodal, $P_{t d}$, and performed a linear fit with two variables:

$$
P_{t d}\left(L_{01}, K_{\mathrm{sym}, 01}\right)=a \times L_{01}+b \times K_{\mathrm{sym}, 01}+c,
$$

The following relation was obtained

$$
\begin{aligned}
P_{t d}\left(L_{01}, K_{\mathrm{sym}, 01}\right)= & 9.59 \times 10^{-3} \times\left[L_{01}-0.343 \times K_{\mathrm{sym}, 01}\right] \\
& -0.328 \mathrm{MeV} \mathrm{fm}^{-3} .
\end{aligned}
$$

This correlation is represented on Fig. 4 where it is compared with corresponding $P_{t d}$ versus $L$ plot. A similar relation has been verified recently by the authors of Ref. 60, although a different slope coefficient is obtained, which they attribute to the different method used to determine the transition point.

\section{Symmetry energy and the pasta phase}

Neutron stars and proto-neutron stars are believed to have in the inner crust a special non-homogeneous matter known as pasta phase. The pasta phase is a frustrated system that arises from the competition between the strong and the electromagnetic interactions $[61,62,63,64,65$, 63 and appears at densities of the order of $0.001-0.1 \mathrm{fm}^{-3}$ [56, 65] in neutral nuclear matter or in a smaller density range [57,66] in $\beta$-equilibrium stellar matter. The basic shapes of these structures (droplets (bubbles), rods (tubes) and slabs for three, two and one dimensions respectively) were first discussed in 61, where the authors joked on the resemblance with lasagna, spaghetti and so on, from which the phase name was chosen. It was shown in [67] that the EOS of the inner crust is particularly sensitive to the density dependence of the symmetry energy, and, therefore, it is expected that the pasta phase structure will depend on it.

In the following we discuss how the density dependence of the symmetry energy affects the pasta phase and the inner-crust structure within a nuclear relativistic mean-field approach. We adopt, in line with many authors, the following simplifying view: for some given conditions (temperature, density, proton fraction or chemical equilibrium) a single geometry will be the physical one. That is, in practice, we compute the free energy of homogeneous matter and the five structures and choose as the physical one that with the smaller free energy. The denser regions (clusters) will form a regular lattice that we study in the Wigner-Seitz (WS) approximation.

First, we will discuss the pasta phase properties within a naive picture that uses the Gibbs conditions of coexisting phases and includes by hand the surface and Coulomb contributions [10,56,57. This description will be denoted by the coexisting phases $(\mathrm{CP})$ method and will allow the identification of the main pasta features that depend on the symmetry energy.

Next, we will present a complete self-consistent calculation of the pasta phase within a relativistic mean field density dependent Thomas-Fermi approach (TF) [56]. What before was described as a two-density system with a sharp interface, is now described as a system with smoothly varying densities. In Ref. 9] we compared the TF and CP approaches and found that the TF method confirms the main trends given by the more naive $\mathrm{CP}$ method, but predicts a wider and richer pasta phase. It is worth emphasizing that other approaches are available to build the pasta phase, each with its advantages and disadvantages. For example, a Hartree-Fock-Bogoliubov (HFB) calculation would allow to include the shell effects, neglected in the RMF approach, however, within this framework only the spherical symmetry could be addressed. For a comparison of HFB and TF results in this context see Ref. [11.

Finally, we will use the TF EOS for the pasta phase as an ingredient to compute the star structure, and we will discuss how the presence of the pasta phase affects the inner crust. 

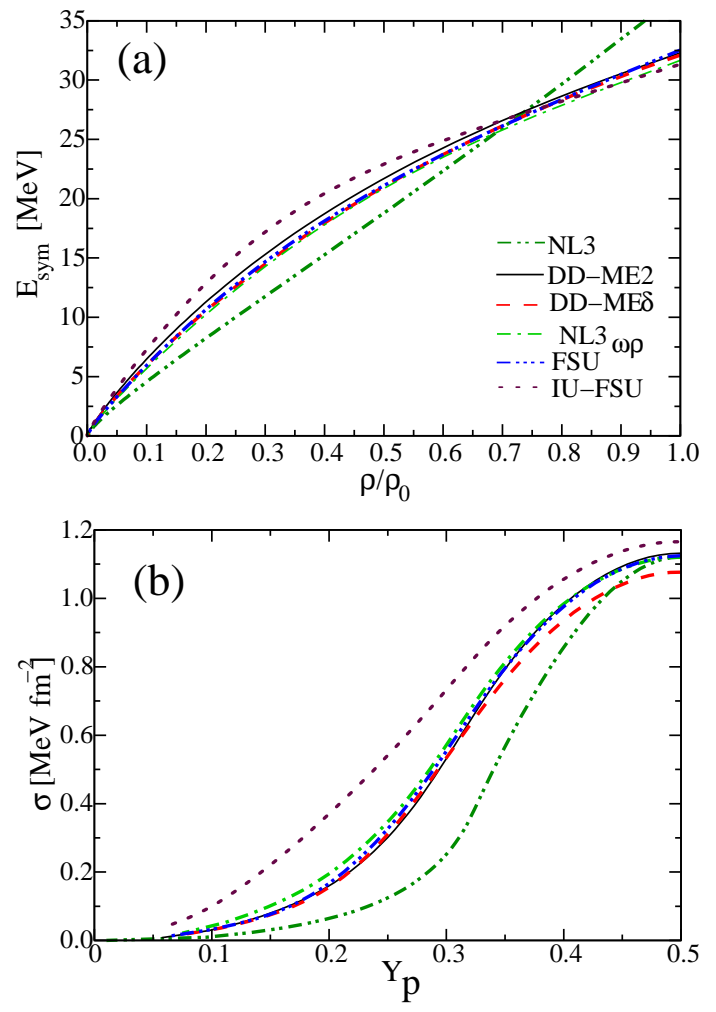

Fig. 5. (Color online) a) Symmetry energy vs density and b) surface tension at $T=0$ vs the proton fraction for NL3, NL3 $\omega \rho$, FSU, IU-FSU, DD-ME2 and DD-ME $\delta$.

\subsection{Surface tension and symmetry energy}

Before entering the discussion of the pasta phase, and in order to compare the models used in this part, namely NL3, NL3 $\omega \rho$, FSU, IU-FSU, DDME2 and DDME $\delta$, we plot in Fig. 5 the symmetry energy vs density, and the surface tension $\sigma$ vs the proton fraction for all of them. The surface tension coefficient $\sigma$ (shown here for $T=0$ ) was obtained in the TF approach along the lines explained in Ref. [10 and it is used in a parametrized form as an input in our CP calculation. The properties of the pasta phase in the CP approach depend crucially on $\sigma$; also in the TF approach, where surface terms are generated selfconsistently, it is a useful guideline to interpret the results.

\begin{tabular}{lcccccc}
\hline \hline & NL3 & NL3 $\omega \rho$ & FSU & IU-FSU & DDME2 & DDME $\delta$ \\
\hline$\rho_{0}$ & 0.148 & 0.148 & 0.148 & 0.155 & 0.152 & 0.152 \\
$J$ & 37.3 & 31.7 & 32.6 & 31.3 & 32.3 & 32.4 \\
$L$ & 118.3 & 55.2 & 60.5 & 47.2 & 51.4 & 52.9 \\
$K_{0}$ & 270.7 & 272.0 & 230.0 & 231.2 & 250.8 & 219.1 \\
\hline \hline
\end{tabular}

Table 1. Properties at saturation: density $\rho_{0}\left(\mathrm{fm}^{-3}\right)$, symmetry energy value $J(\mathrm{MeV})$ and slope $L(\mathrm{MeV})$, and incompressibility $K_{0}(\mathrm{MeV})$, for the models discussed in Sec. 4.

To help the discussion of this section, we present in Table 1 the isovector properties at saturation predicted by the six models considered here. As shown in the table the six models predict very similar values for the symmetry energy at saturation, namely, $J$ varies between 31.3 and 32.6 MeV, except for NL3 that has a quite high value, 37.3 $\mathrm{MeV}$. However, there is a larger dispersion of the symmetry energy slope at saturation, $L$, with values that go from 47.2 MeV (IU-FSU), to 118.3 MeV for NL3.

The properties of the pasta will reflect these facts, with IU-FSU and NL3 behaving in a quite different way, while all the other models show similar results. The slope $L$ has a direct influence on the surface tension and surface thickness of the clusters. A smaller $L$ corresponds generally to a larger surface tension and smaller neutron skin thickness 44, as can be seen by comparing the surface tensions of the above models. The decrease of the surface tension with the slope $L$ may be understood from the fact that the neutron pressure at a density close to $0.1 \mathrm{fm}^{-3}$, a typical density at the nucleon surface, is essentially proportional to the slope $L$ 6, 68. Therefore, a larger value of $L$ will favor neutron drip and a smaller surface tension, i.e., particles at the surface are not so tightly bound to the nucleus.

\subsection{Pasta phase}

\subsubsection{Coexisting phases (CP) method}

As first approximation, the pasta phase is calculated within the CP method [56]. In this section we focus on nuclear matter with a fixed proton fraction $Y_{p}$ and impose charge neutrality by setting $\rho_{e}=\rho_{p}$. However, the same scheme could be applied to $\beta$-equilibrium stellar matter: in this case, the species fractions would be defined by the conditions of chemical equilibrium and charge neutrality.

As in 56 63, for a given total density $\rho$, the pasta structures are built with different geometrical forms in a background nucleon gas. This is achieved by calculating from the Gibbs conditions $P^{I}=P^{I I}, \mu_{i}^{I}=\mu_{i}^{I I}$ where I and II label the high and low density phase respectively, the density and the proton fraction of the pasta and of the background gas. The density of electrons is considered uniform. The total pressure and total energy density of the system are given, respectively, by $P=P^{I}+P_{e}$ and

$$
\mathcal{E}=f \mathcal{E}^{I}+(1-f) \mathcal{E}^{I I}+\mathcal{E}_{e}+\mathcal{E}_{\text {surf }}+\mathcal{E}_{\text {Coul }},
$$

where $f$ is the volume fraction of phase $\mathrm{I}$, the proton fraction can be obtained from

$$
f \rho_{p}^{I}+(1-f) \rho_{p}^{I I}=Y_{p} \rho,
$$

and $\mathcal{E}_{e}, \mathcal{E}_{\text {surf }}$ and $\mathcal{E}_{\text {Coul }}$ denote electron, surface and Coulomb energy densities. By minimizing $\mathcal{E}_{\text {surf }}+\mathcal{E}_{\text {Coul }}$ with respect to the size of the droplet/bubble, rod/tube or slab we get 63 . $\mathcal{E}_{\text {surf }}=2 \mathcal{E}_{\text {Coul }}$, and

$$
\mathcal{E}_{\text {Coul }}=\frac{2 F}{4^{2 / 3}}\left(e^{2} \pi \Phi\right)^{1 / 3}\left(\sigma D\left(\rho_{p}^{I}-\rho_{p}^{I I}\right)\right)^{2 / 3}
$$




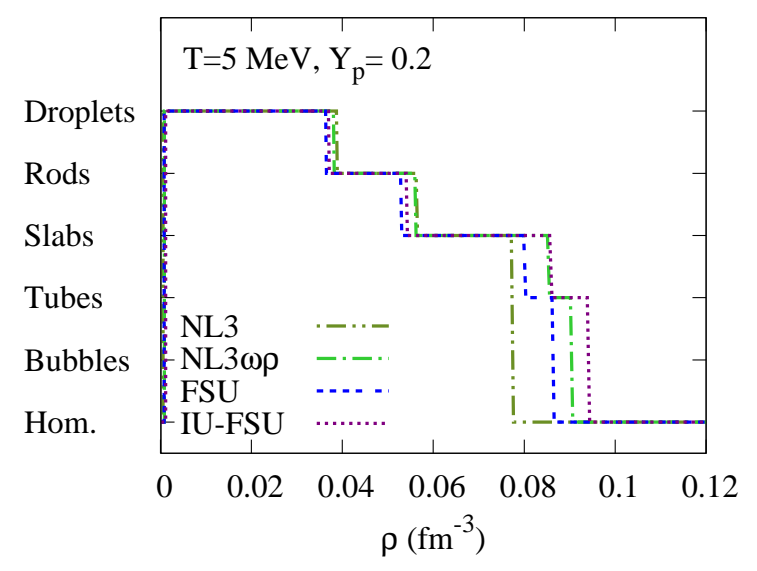

Fig. 6. (Color online) Pasta phases in the CP method for $T=5 \mathrm{MeV}$ and $Y_{p}=0.2$. Results for NL3, NL3 $\omega \rho$, FSU, IU-FSU.

where $F=f$ for droplets and $F=1-f$ for bubbles, $\sigma$ is the surface energy coefficient, $D$ is the dimension of the system and $\Phi$ is the geometric factor:

$$
\Phi=\left(\frac{2-D F^{1-2 / D}}{D-2}+F\right) \frac{1}{D+2}, D=1,2,3 .
$$

In the following discussion the parameter sets for the models NL3, NL3 $\omega \rho$, FSU and IU-FSU will be considered and the effect of the symmetry energy on the pasta phase discussed. One expects, generally speaking, two types of effects: a smaller $L$ corresponds to a larger surface tension for asymmetric matter [10] and a larger $J$ leads to a more isospin-symmetric liquid phase. In models with a larger surface tension the pasta phase sets in at higher densities and neutron drip is unfavored, giving rise to a lower density background gas.

In Fig. 6] the range of the different pasta phases for matter with a proton fraction $Y_{p}=0.2$ and temperature $T=5 \mathrm{MeV}$ is plotted for these four models. Some comments are in order: for the proton fraction considered no model presents the bubble configuration, and for NL3 the tube configuration is also missing; the onset of the rod and slab configurations are quite model independent, while the transition to the core reflects the symmetry energy behavior, in particular, the transition density is smaller for larger values of $L$, as discussed above.

In Fig. 7we show some pasta properties, mainly for the droplet phase. Let us denote by $A_{\text {drop }}$ the number of nucleons belonging to a droplet (the type of structure that appears at the lowest densities in the non-homogeneous phase) and by $Z_{\text {drop }}$ its charge content. Notice that we adopt the prescription to define both quantities as excesses with respect to the background nucleon gas. In the left panel we display the results for a) $A_{\text {drop }}$ and b) $Z_{\text {drop }}$ for the above parametrizations. The onset of the droplet phase is characterized by a discontinuity on the number of nucleons inside the cluster: a minimum number of nucleons is necessary to compensate the surface energy, which is larger for models with a smaller $L$. It should be referred, however, that in a TF calculation, where the surface energy is calculated self-consistently, this discontinuous behavior will not occur. A change in the isovector channel of the model NL3 as in NL3 $\omega \rho$ leads to a large effect on the number of nucleons in the droplet, increasing this number to more than the double. In fact, a smaller symmetry energy slope corresponds to a larger surface energy and neutrons do not drip out so easily. The number of nucleons obtained within NL3 $\omega \rho$ is consistent with the results of 69] within a statistical model. The other two models, FSU and IU-FSU, also present larger nuclei than NL3, the heaviest ones corresponding to the model IU-FSU, which bears the smallest slope $L$.

The radius of the Wigner Seitz cell together with the cluster radius is plotted in the middle panel of Fig. 7 as a function of density. The ordering of the radii obtained in the different parametrizations reflects perfectly the ordering of their surface tensions [10, that, in turn, is closely linked to the symmetry energy density dependence. NL3 has by far the smallest surface energy at $Y_{p}=0.2$, while IU-FSU has the largest: correspondingly, NL3 has the smallest Wigner Seitz cell and droplets and IU-FSU the largest ones.

In the right panel of Fig. 7 the ratio $Z_{\text {drop }} / A_{\text {drop }}$ is plotted as a function of density. This ratio decreases with density and is model dependent. A decrease of the proton fraction of the clusters with density was also obtained in [70]. The models with a smaller symmetry energy slope have smaller proton fractions. A smaller slope implies that neutrons drip out of the cluster with more difficulty giving rise to neutron richer clusters. Also, a smaller slope corresponds to a smaller $J$, and, thus, a smaller $L$ favors less symmetric clusters.

In summary, within the coexistence method we have shown that models with a smaller symmetry energy slope have larger clusters with a smaller proton fraction and larger Wigner-Seitz cells. We will next discuss the predictions of a Thomas Fermi calculation of the pasta phase [11, which generally agree with the above conclusions.

\subsubsection{Thomas-Fermi (TF) approach}

In the Thomas-Fermi approximation of the non-uniform npe matter, the fields are assumed to vary slowly so that the baryons can be treated as moving in locally constant fields at each point 63.56. We obtain the finite temperature semiclassical TF approximation based on the density functional formalism [10] and start from the grand canonical potential density:

$\omega=\omega\left(\left\{f_{i+}\right\},\left\{f_{i-}\right\}, \sigma_{0}, \omega_{0}, \rho_{0}, \delta_{0}\right)=\mathcal{E}_{t}-T \mathcal{S}_{t}-\sum_{i=p, n, e} \mu_{i} \rho_{i}$

where $\left\{f_{i+}\right\}\left(\left\{f_{i-}\right\}\right), i=p, n, e$ stands for the protons, neutrons and electrons positive (negative) energy distribution functions and $\mathcal{S}_{t}=\mathcal{S}+\mathcal{S}_{e}, \mathcal{E}_{t}=\mathcal{E}+\mathcal{E}_{e}$ are the total entropy and energy densities respectively [9]. The equations 

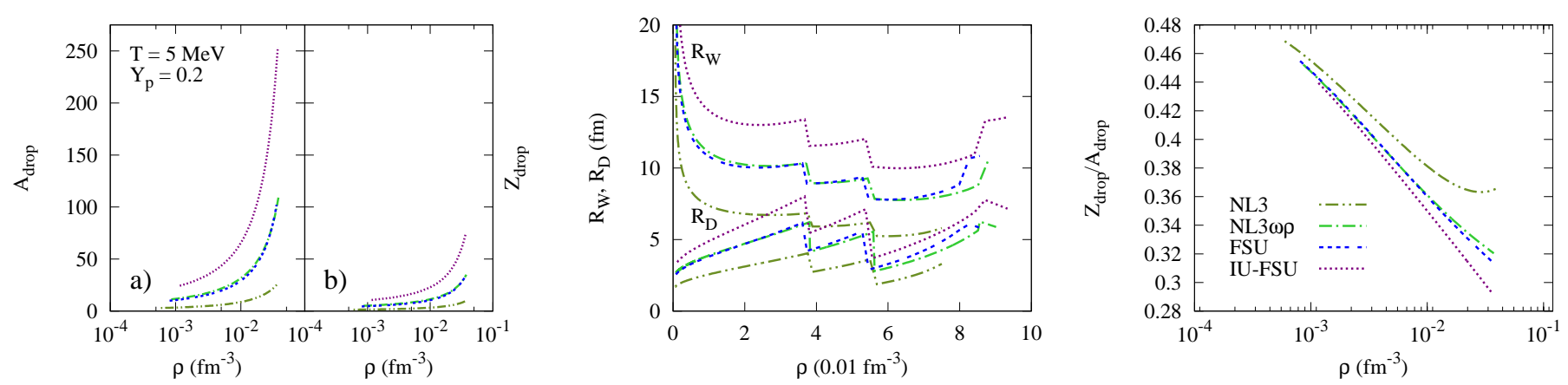

Fig. 7. (Color online) Properties of pasta obtained in the CP method, at $T=5 \mathrm{MeV}$ and $Y_{p}=0.2$. Results for NL3, NL3 $\omega \rho$, FSUand IU-FSU parametrizations. Left: The number of a) nucleons $A$ and b) protons $Z$ in a droplet. Middle: cluster and Wigner Seitz cell radius. Right: The ratio $Z / A$ in the droplet phase.

of motion for the meson fields (see Ref. [56]) follow from the variational conditions:

$$
\frac{\delta}{\delta \sigma_{0}(\mathbf{r})} \Omega=\frac{\delta}{\delta \omega_{0}(\mathbf{r})} \Omega=\frac{\delta}{\delta \rho_{0}(\mathbf{r})} \Omega=\frac{\delta}{\delta \delta_{0}(\mathbf{r})} \Omega=0,
$$

where $\Omega=\int d^{3} r \omega$.

The numerical algorithm for the description of the neutral npe matter at finite temperature is a generalization of the zero temperature case which was discussed in detail in 9,56 . The Poisson equation is always solved by using the appropriate Green function according to the spatial dimension of interest and the Klein-Gordon equations are solved by expanding the meson fields in a harmonic oscillator basis with one, two or three dimensions based on the method presented in 9, 56.

We next present results for the pasta phase of $\beta$-equilibrium matter obtained within a $\mathrm{TF}$ calculation at $\mathrm{T}=0$. Due to the $\beta$-equilibrium condition the proton fraction is very small and only three different shapes occur: droplet, rod and slab. The transition densities between the shapes are compared in Fig. 8 for the six models mentioned above. All of the three shapes appear in the inner crust except for NL3, which only predicts the existence of droplets. In fact, in 67] it was shown that models with a large $L$, like NL3, would not predict the existence of non-droplet pasta shapes in $\beta$-equilibrium matter. As discussed before, the slope $L$ defines the crust-core transition within models in the same framework. It is seen, however, that although IU-FSU has the largest crust-core transition density to the core, it also has the smallest transition density to the rod and slab configurations. This behavior probably reflects the large surface energy of IU-FSU that favors smaller surface to volume geometries.

In Fig. 9 we show the neutron density at the cell center and at the cell border (left panel), the cluster proton fraction at the cluster center (middle panel) and the total atomic number of a cluster (right panel) [11. As in the $\mathrm{CP}$ method, also here the background nucleon gas is subtracted when defining the cluster properties $Y_{\mathrm{p}, \mathrm{drop}}$ and A. Notice that the number of nucleons belonging to a cluster or to a cell ( $\mathrm{A}, \mathrm{N}$ and $\mathrm{Z})$, are univocally determined by the calculation only in the case of droplets. For the slab and

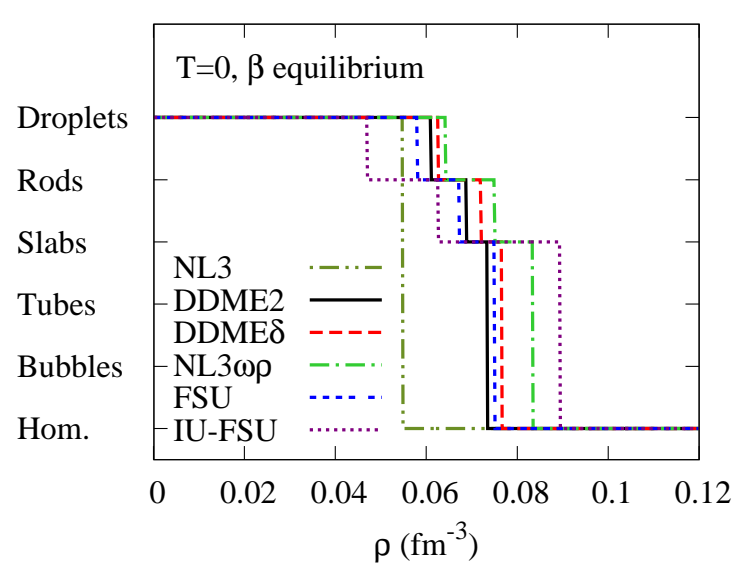

Fig. 8. (Color online) Pasta phases in $\mathrm{TF}$ method at $\mathrm{T}=0$ in $\beta$-equilibrium matter. Results for NL3, NL3 $\omega \rho$, FSU, IUFSU, plus two density dependent hadronic parametrizations, DDME2 and DDME $\delta$.

rod phases, by construction, the problem is only solved in one or two dimensions. The values of $\mathrm{A}, \mathrm{Z}$ and $\mathrm{N}$ for these shapes were obtained assuming representative sizes for the rod length and for the slab cross-section [11.

These results are coherent with the ones calculated within the coexisting phases method. The density of dripped neutrons is smallest for IU-FSU, the model with the smallest slope $L$. Moreover, IU-FSU (NL3) has the largest (smallest) number of nucleons in the clusters, corresponding to the smallest (largest) slope $L$, and at the cluster center the proton fraction is largest for models with the largest symmetry energy $J$.

In Fig. 10 we show some properties of the WS cells: the proton (a) and neutron (b) content of each cell ( $\mathrm{N}$ and $\mathrm{Z}$ ), and the cell radius (c).

The properties of the models used are reflected on the cluster structure. A small symmetry energy slope $L$ gives rise to larger cells, with a larger proton and neutron number, while the opposite occurs for a large $L$. Models with 

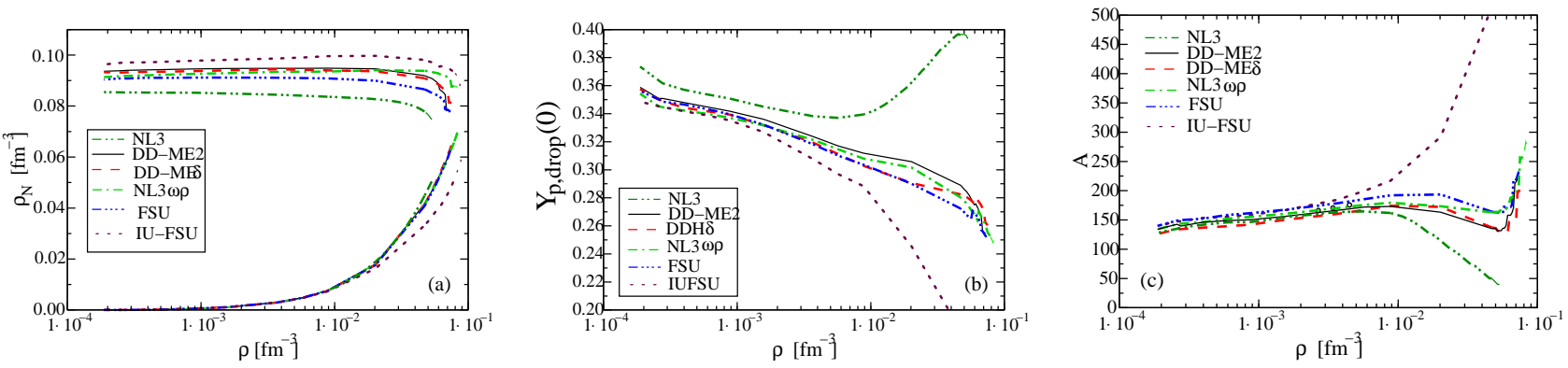

Fig. 9. (Color online) Neutron density at the cell center (upper curves) and border (lower curves) (a), cluster proton fraction at the cluster center (b) and the total atomic number of a cluster (c) for the same models as in Fig. 8
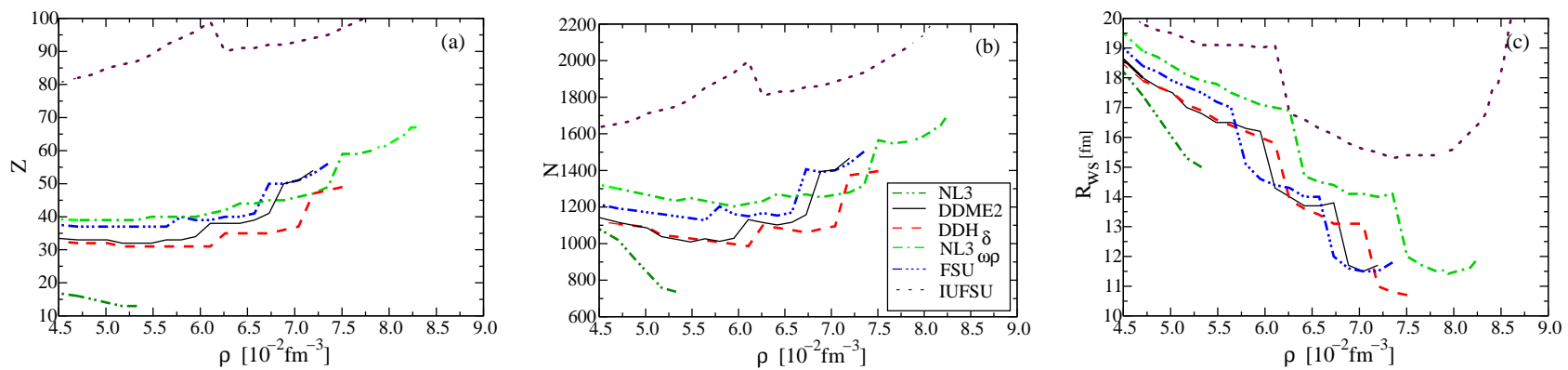

Fig. 10. (Color online) a) Proton number, b) neutron number and c) radius of a WS cell in the pasta phase regions. Same models as in Fig. 8 .

a similar symmetry energy $(\sim 31-32 \mathrm{MeV})$ and slope $L$ $(\sim 50-60 \mathrm{MeV})$ at saturation density behave in a similar way, both in the droplet phase and the non-droplet pasta phase regions. On the other hand, models like NL3, with a very large symmetry energy and slope $L$, and IU-FSU, with a quite small $L$, have quite different behaviors. NL3 does not present any non-droplet pasta phase in the inner crust of $\beta$-equilibrium matter, predicts the smallest proton and neutron numbers and the Wigner-Seitz radius in almost all the inner crust range of densities.

All of the models, except NL3, predict the existence of slab like configurations in $\beta$-equilibrium matter. These lasagna-like structures may have an important contribution to the specific heat of the crust [12].

\subsection{Inner crust structure}

In this section we analyse how the EOS at subsaturation densities affects the inner-crust extension.

The Tolman-Oppenheimer-Volkov (TOV) equations are solved to determine the density profile of neutron stars with masses $1,1.44,1.6 M_{\odot}$. These are stars with representative masses: the lowest one is smaller than the smallest neutron stars detected until now, $1.44 M_{\odot}$ is the mass of the Hulse-Taylor pulsar, and the largest mass is chosen to be smaller than the maximum mass described by FSU. Besides these three values, the TOV equations are also solved for the maximum mass star. The stellar matter EOS's are built according to the following scheme 71: a) the EOS in the core is obtained including only nucleonic degrees of freedom, electrons and muons, solving the equations for the meson fields in the mean-field approximation and imposing both $\beta$-equilibrium and charge neutrality; b) for the outer crust, the BPS (Baym-Pethick-Sutherland) EOS 72 is considered; c) the inner crust, corresponding to the range of densities between the neutron drip $\left(\sim 2 \times 10^{-4}\right.$ $\mathrm{fm}^{-3}$ ) and the crust-core transition, is obtained from the TF calculation of $\beta$-equilibrium non-homogeneous matter [10, 11, 56, 57.

In Table 2 we display some of the features of the inner crust structure according to different models. All models considered have a slab and a rod phase which together define the non-droplet pasta extension, except for NL3. For this model the inner crust is only formed by droplets in a neutron gas background. For the sake of readability, some of the results given in Table 2 are plotted in Fig. 11 and 12. In Fig. 11 the thickness of the crust (full symbols) and inner crust (empty symbols) are given in the left panel, the thickness of the total non-droplet pasta phase (full symbols) and the slab phase (empty symbols) are plotted in the middle panel, and the fraction of the inner crust with respect to the total crust is given in the right panel. The different models are ordered according to the magnitude of the slope $L$, which increases from left to right. In Fig. 12 we represent instead the crust profile, identifying the transition between the different configurations with marks (black lines and symbols). In the same figure, it is also shown the crust profile calculated with an EOS obtained joining the BPS EOS directly to the homogeneous stellar matter EOS (red dashed lines). In this last case the transition from the BPS to the homogeneous matter EOS is shown by a red full point. 

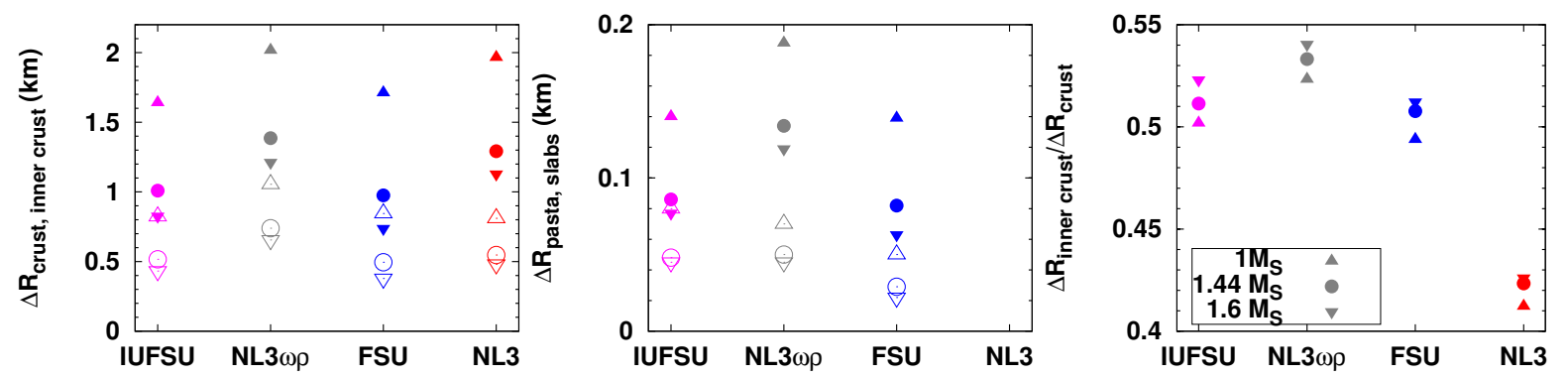

Fig. 11. (Colors online) Left: thickness of the crust (full symbols) and of the inner crust (empty symbols). Middle: thickness of the non-droplet pasta (full symbols) phase and of the slab (empty symbols) phase. Right: fraction of the crust occupied by the inner crust.
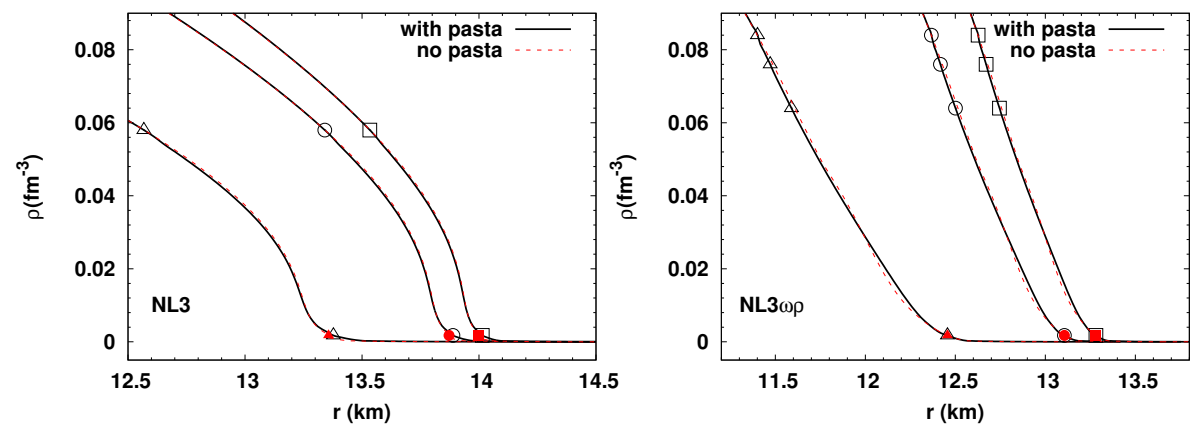

Fig. 12. (Colors online) Crust of neutron stars with a mass equal to 1.0, 1.44 and $1.6 M_{\odot}$ obtained for different nuclear models (solid lines). The empty symbols stay at the BPSdroplet, droplet-rod, rod-slab, slab-
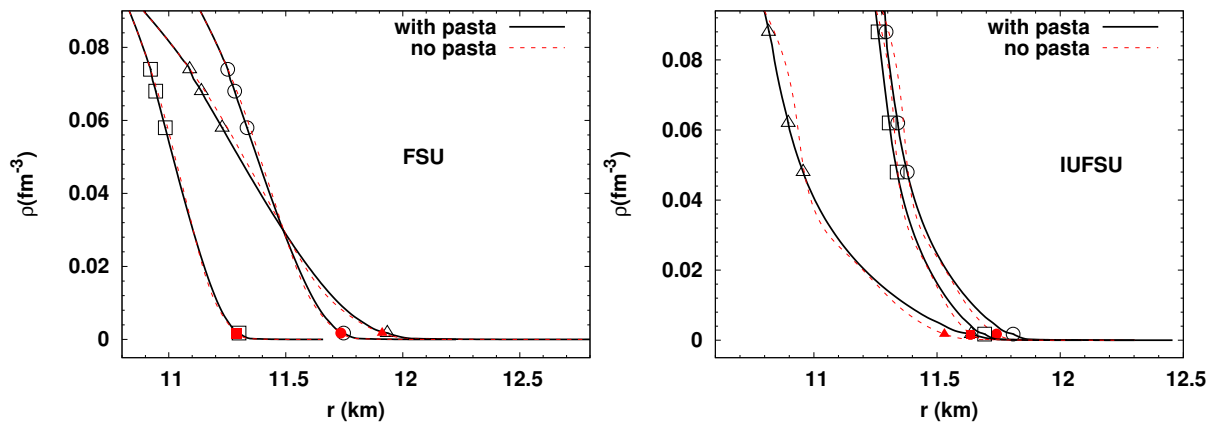
homogeneous transition for $1.0 M_{\odot}$ (circle), $1.44 M_{\odot}$ (triangle), $1.6 M_{\odot}$ (square). For comparison, the profiles obtained without pasta (dashed) are also shown. The full symbols denote the BPS-homogeneous matter transition in this case.

The extension of the total crust is mainly defined by the incompressibility of the EOS (cfr Table 1). However, the fraction of the crust occupied by the inner crust is of the order of $50 \%$ or less and depends on the symmetry energy. This quantity increases with the star mass, resulting in a difference of $\sim+5 \%$ between stars of mass 1.6 and $1.0 M_{\odot}$.

The strong differences existing between NL3 and NL3 $\omega \rho$ allow us to identify the effect of the symmetry energy, since these two models only differ with respect to the density dependence of the symmetry energy. It has already been shown that there is an anti-correlation between the crustcore transition density and the slope $L$ at saturation 6. 8] and, therefore, it could be expected a larger crust for NL3 $\omega \rho$. We point out, however, that this correlation does not exist with the total crust thickness, but only with the inner crust. In a $1.0 M_{\odot}$ star the non-droplet pasta extension is smaller than $200 \mathrm{~m}$. Generally the stars with smaller mass have smaller relative pasta phases. The slab fraction corresponds to $\sim 35 \%$ of the total pasta phase for all the models, apart from IU-FSU, where it is almost $60 \%$. The different behavior of IU-FSU is mainly due to the small value of the symmetry energy slope at subsaturation densities, which affects the surface tension giving quite high surface tension for different proton fractions, see 11. A large surface tension favors the slab geometry with respect to the rod geometry. On the contrary, a smaller surface tension favors the formation of droplets, clusters with the largest surface for the same volume, in a larger density range.

In Fig. 12 we have plotted the last $\sim 2 \mathrm{~km}$ of the star profile closer to the surface. A larger mass corresponds to a steeper profile as expected, due to the larger gravitational force. For NL3 and NL3 $\omega \rho$, both with a large incompressibility, the star with the larger mass has the inner crust at a larger distance from the center. In the case of FSU and IU-FSU there is a larger concentration of mass at the center because the EOS is softer, and the crust is pushed more strongly towards the center of the star: this explains why for IU-FSU the profiles of the 1.44 


\begin{tabular}{lcccccc}
\hline \hline $\begin{array}{l}M \\
\left(M_{\odot}\right)\end{array}$ & $\begin{array}{c}\epsilon_{c} \\
\left(\mathrm{fm}^{-4}\right)\end{array}$ & $\begin{array}{c}R_{h s} \\
(\mathrm{~km})\end{array}$ & $\begin{array}{c}R_{s r} \\
(\mathrm{~km})\end{array}$ & $\begin{array}{c}R_{r d} \\
(\mathrm{~km})\end{array}$ & $\begin{array}{c}R_{d B P S} \\
(\mathrm{~km})\end{array}$ & $\begin{array}{c}R \\
(\mathrm{~km})\end{array}$ \\
\hline & & & & & & \\
1.00 & 1.81 & 11.09 & 11.14 & 11.23 & 11.93 & 12.80 \\
1.44 & 3.07 & 11.25 & 11.28 & 11.33 & 11.75 & 12.28 \\
1.60 & 4.39 & 10.92 & 10.94 & 10.99 & 11.30 & 11.66 \\
1.66 & 7.04 & 10.27 & 10.29 & 10.32 & 10.56 & 10.84 \\
& & & & & & \\
& & & IU-FSU & & & \\
1.00 & 1.78 & 10.82 & 10.90 & 10.96 & 11.64 & 12.46 \\
1.44 & 2.61 & 11.29 & 11.34 & 11.38 & 11.81 & 12.30 \\
1.60 & 3.18 & 11.26 & 11.31 & 11.34 & 11.69 & 12.09 \\
1.80 & 6.69 & 10.48 & 10.50 & 10.52 & 10.74 & 11.00 \\
& & & & & & \\
& & & NL3 & & & \\
1.00 & 1.12 & 12.57 & - & - & 13.38 & 14.53 \\
1.44 & 1.39 & 13.34 & - & - & 13.89 & 14.63 \\
1.60 & 1.49 & 13.53 & - & - & 14.01 & 14.66 \\
2.78 & 4.42 & 12.78 & - & - & 13.12 & 13.29 \\
& & & & & & \\
& & & NL3 $\omega \rho$ & & & \\
1.00 & 1.28 & 11.40 & 11.47 & 11.59 & 12.46 & 13.42 \\
1.44 & 1.52 & 12.37 & 12.42 & 12.50 & 13.11 & 13.75 \\
1.60 & 1.62 & 12.63 & 12.67 & 12.75 & 13.28 & 13.84 \\
2.68 & 4.62 & 12.49 & 12.50 & 12.52 & 12.70 & 12.87 \\
\hline \hline
\end{tabular}

Table 2. Central energy density, distance to the center of the star at the phase transitions: homogeneous matter-slab phase $\left(R_{h s}\right)$, slab phase-rod phase $\left(R_{s r}\right)$, rod-phase-droplet phase $\left(R_{r d}\right)$, droplet phase-outer crust $\left(R_{d B P S}\right)$ and radius of a 1.0 , 1.4, and 1.6 $M_{\odot}$ star for several EOS, for different models. For each model, the maximum mass configuration is also shown.

and $1.6 M_{\odot}$ stars are almost coincident, and for FSU the profiles of the 1.0 and $1.44 M_{\odot}$ stars cross, while the crust of the $1.6 M_{\odot}$ one has the smallest distance to the star center. One interesting conclusion is that taking into account the correct description of the inner crust in the total stellar EOS is more important for the softer EOS and with smaller slopes $L$. However, on the whole, using the BPS EOS for the outer crust and an EOS of homogeneous stellar matter for the inner crust and core gives good results for the stellar profiles.

In 73 the effect of the nuclear pasta on the crustal shear phenomena was studied. In particular, two limits have been considered, namely the pasta as an elastic solid and as a liquid. In the first case the shear modulus is calculated from the crust-core transition while in the second case at the transition from the droplet to the pasta phase. For models with no pasta phase, as NL3, there is no difference between these two pictures. However, models with a symmetry energy slope $L$ below $80 \mathrm{MeV}$ have a pasta phase and the ratio shear modulus to pressure may be as high as two times larger if the first picture is considered for $L=40 \mathrm{MeV}$.

\section{Symmetry energy and the strangeness content of a neutron star}

For stellar matter with hyperonic degrees of freedom, as the one described in this section, the electromagnetic-field is switched off, the sum over nucleons in Eq. (9) is replaced by a sum over the octet of lightest baryons (n, p, $\Lambda, \Sigma^{-}$, $\left.\Sigma^{0}, \Sigma^{+}, \Xi^{-}, \Xi^{0}\right)$, and the couplings of the mesons to the baryons are baryon dependent. Due to the Pauli principle the nucleon Fermi energy increases and, if the Fermi energy of nucleons becomes larger than the hyperon masses, energy and pressure are lowered by conversion of some nucleons into hyperons. This softens the equation of state and has some direct consequences on the properties of compact stars: maximum star masses become smaller and neutrino fractions in neutrino trapped matter are larger.

To fix the model, we need to define the couplings $g_{i j}$, where $i$ is any meson and $j$ any baryon of the octet. For the nucleonic sector, we use the IU-FSU [47] and TM1 43. parametrizations. The latter is a parametrization that satisfies the heavy-ion flow constraints for symmetric matter at $2-3 \rho_{0}$ 74. To better understand the effect of the symmetry energy on the strangeness content, the mass and radius of the stars, we will consider a modified version of IU-FSU with $\Lambda_{\omega}$ as a free parameter. Analogously, for TM1, we will discuss a modified version obtained including a non-linear $\omega-\rho$ term $(\mathrm{TM} 1 \omega \rho)$ that will allow to change the density dependence of the symmetry energy, as presented in [16], when $\Lambda_{\omega}$ runs from 0 (TM1) to 0.03 . For the slope of the symmetry energy at saturation density we have the following values: $\Lambda_{\omega}=0(L=110 \mathrm{MeV})$, $\Lambda_{\omega}=0.01(L=80 \mathrm{MeV}), \Lambda_{\omega}=0.02(L=70 \mathrm{MeV})$ and $\Lambda_{\omega}=0.03(L=55 \mathrm{MeV})$.

For the hyperons, we consider two different sets of hyperon-meson couplings, that we name $\mathrm{A}$ and $\mathrm{B}$. Within the coupling set A [75] the $\omega$ and $\rho$ meson-hyperon coupling constants are obtained using the $\mathrm{SU}(6)$ symmetry: $\frac{1}{2} g_{\omega \Lambda}=\frac{1}{2} g_{\omega \Sigma}=g_{\omega \Xi}=\frac{1}{3} g_{\omega N}, \frac{1}{2} g_{\rho \Sigma}=g_{\rho \Xi}=g_{\rho N}, g_{\rho \Lambda}=$ 0 , where $N$ means 'nucleon' $\left(g_{i N} \equiv g_{i}\right)$. The coupling constants $g_{\sigma Y}$ of the hyperons with the scalar meson $\sigma$ are constrained by choosing the hypernuclear potentials in nuclear matter to be consistent with hypernuclear data [76]. Namely, we impose (see Ref. [76]) $U_{\Lambda}=-28 \mathrm{MeV}, U_{\Sigma}=$ $30 \mathrm{MeV}, U_{\Xi}=-18 \mathrm{MeV}$, being $U_{j}=x_{\omega j} U_{\omega}-x_{\sigma j} U_{\sigma}$ where $x_{i j} \equiv g_{i j} / g_{i}, U_{\omega} \equiv g_{\omega} \omega_{0}$ and $U_{\sigma} \equiv g_{\sigma} \sigma_{0}$ are the nuclear potentials for symmetric nuclear matter at saturation.

In order to show how results are sensitive to the hyperon couplings we consider a quite different set of couplings proposed in 45, set B, with $x_{\sigma Y}=0.8$ and equal for all the hyperons. The fraction $x_{\omega Y}$ is determined using $U_{Y}=-28 \mathrm{MeV}$ for all the hyperons. For the hyperon$\rho$-meson coupling we consider $x_{\rho Y}=x_{\sigma Y}$. This choice implies that the interaction of all hyperons in symmetric nuclear matter is attractive, and is restricted by acceptable maximum mass star configurations.

The onset density of the nucleon Direct Urca (DU) process is plotted as a function of the slope $L$ for the IU-FSU and modified versions in Fig. 13. The effect of 


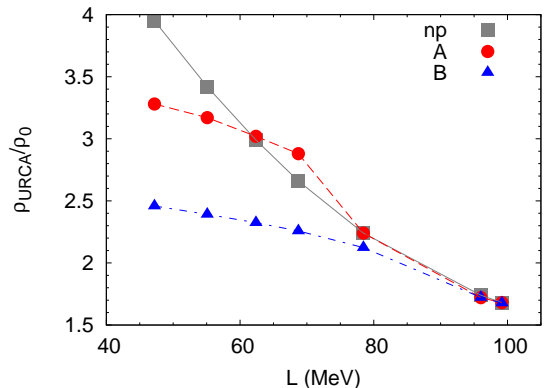

Fig. 13. (Color online) Onset density of the direct Urca process in stellar matter for nucleonic matter (gray squares), hyperon coupling set A (red circles) and hyperon coupling set $\mathrm{B}$ (blue triangles) for IU-FSU and modified versions with $L=47-99 \mathrm{MeV}$.

the symmetry energy and the hyperon interaction on the DU onset density can be summarized as follows a) for non-strange matter the larger the slope $L$ the smaller the neutron-proton asymmetry above the saturation density and, therefore, the smaller the DU onset density; b) generally, for a low value of $L$ the presence of hyperons decreases the onset density. This is always true if the hyperon onset occurs with a negatively charged hyperon because the proton fraction increases. However, if the hyperon onset occurs with a neutral hyperon, both the proton and the neutron fractions decrease and it is the net effect that defines the behavior.

In Fig. 14 the strangeness fraction $\left(f_{s}=\sum_{j}\left|s_{j}\right| n_{j} / 3 n_{B}\right.$ with $s_{j}$ and $n_{j}$ the strangeness, partial density of the baryon $j$ and $n_{B}$ the total baryonic density) for IU-FSU and the modified IU-FSU model with $L=99 \mathrm{MeV}$ is plotted as a function of density. A smaller symmetry energy slope hinders the formation of hyperons because it gives rise to a softer EoS. The conditions for the onset of hyperons depend on the charge of the hyperon and on the hyperon interaction: $\Lambda$ is the first hyperon to appear with set $\mathrm{A}$ and occurs at larger densities for a smaller slope $L$, on the contrary, with set $\mathrm{B}, \Sigma^{-}$will occur first and at smaller densities for smaller values of $L[13$.

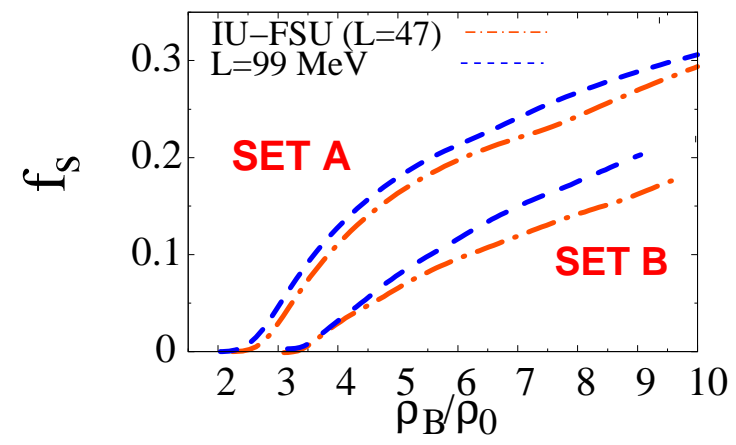

Fig. 14. (Color online) Strangeness fraction with the hyperonmeson coupling sets A and B for IU-FSU $(L=47 \mathrm{MeV})$ and a modified version with $L=99 \mathrm{MeV}$.

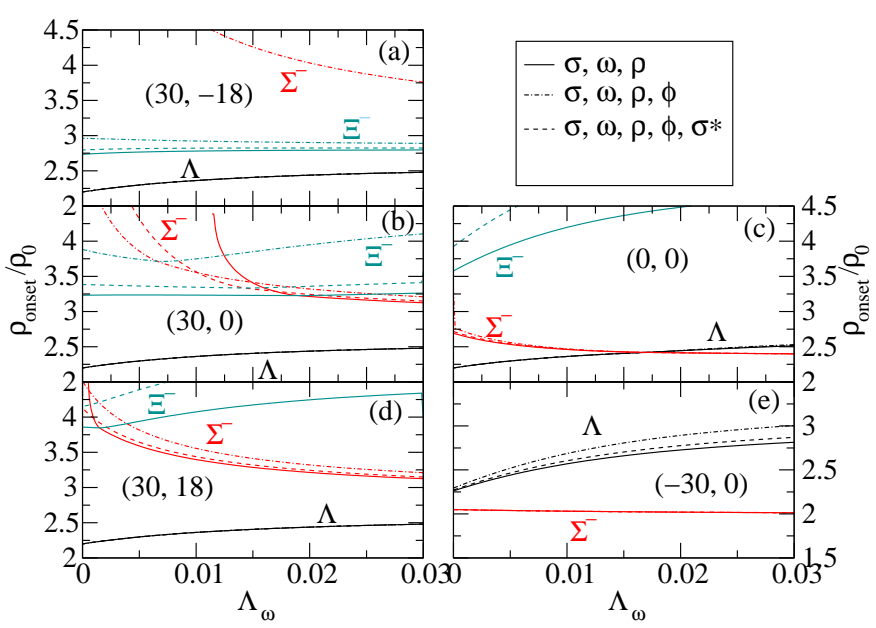

Fig. 15. (Color online) Onset of the $\Lambda, \Sigma^{-}$and $\Xi^{-}$hyperons as a function of the parameter $\Lambda_{\omega}$, with $U_{\Lambda}=-28 \mathrm{MeV}$ and several values of $U_{\Sigma}$ and $U_{\Xi}$ in symmetric nuclear matter at saturation. The limiting values of $\Lambda_{\omega}, 0$ and 0.03 , correspond to $\mathrm{L}=110 \mathrm{MeV}$ and $55 \mathrm{MeV}$ respectively. The pair of values given in each graph refers to $\left(U_{\Sigma}, U_{\Xi}\right)$. Results obtained for the modified TM1 model and extended set A.

From now on, we focus on the TM1 parametrization, and, for the hyperon couplings, on the set A. However, we allow some variations in it. In fact, while the binding of the $\Lambda$ in symmetric nuclear matter is well settled experimentally, the binding values of the $\Sigma^{-}$and $\Xi^{-}$ still have a lot of uncertainties [77]. We, therefore, enlarge the set A by allowing $U_{\Xi}=-18,0,+18 \mathrm{MeV}$ and $U_{\Sigma}=-30,0,30 \mathrm{MeV}$. Finally, we also consider the inclusion of the strange mesons $\sigma^{*}, \phi$ to take into account the YY interactions. According to recent experimental $\Lambda-\Lambda$ hypernuclear data, the $\Lambda-\Lambda$ interaction is only weakly attractive 78 . The effect of the small attractiveness of the hyperon-hyperon coupling will be considered by choosing a) a weak $g_{\sigma^{*} Y}$ coupling according to Eq. 5 of $[79$; b) the extreme value $g_{\sigma^{*} Y}=0$. In both cases, the $\phi$ meson couplings are fixed according to $2 g_{\phi \Lambda}=2 g_{\phi \Sigma}=g_{\phi \Xi}=$ $-\frac{2 \sqrt{2}}{3} g_{\omega N}$.

As mentioned above, the symmetry energy also affects the onset of hyperons. In Fig. 15 it is shown that the different hyperons are affected in a different way by the symmetry energy. In this figure we plot the onset of the $\Lambda, \Sigma^{-}$and $\Xi^{-}$as a function of the coupling $\Lambda_{\omega}$, where $\Lambda_{\omega}=0(0.03)$ corresponds to $L=110(55) \mathrm{MeV}$. It is seen that the onset of $\Sigma^{-}$always decreases with the decrease of $L$, due to its larger isospin. On the other hand, the onset of $\Lambda$ occurs at larger densities. The $\Xi^{-}$is never the first hyperon to appear due to its large mass, but, according to the attractiveness of its potential in nuclear matter, it can appear as the second hyperon. If the repulsiveness of the $\Sigma^{-}$in nuclear matter is confirmed we may expect that the $\Lambda$ is the first hyperon to set on and, therefore, with a smaller slope $L$ the onset of strangeness occurs at larger densities. However, if the optical potential of the $\Sigma^{-}$in nuclear matter is only slightly repulsive there may be a 


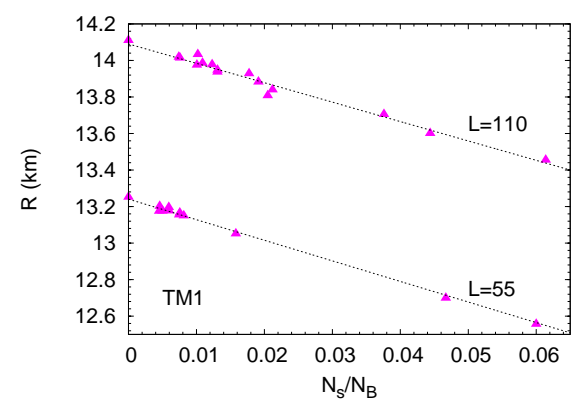

Fig. 16. (Color online) Radius of a star with mass $1.67 M_{\odot}$ as a function of the strangeness content, for TM1 with $L=110$ $\mathrm{MeV}$ (top line with slope $-11.27 \pm 4 \% \mathrm{~km}$ ) and TM1 with $\omega \rho$ term and $L=55 \mathrm{MeV}$ (bottom line with slope $-10.62 \pm 1 \%$ $\mathrm{km}$ ). For a given L, each point corresponds to a choice for the hyperon couplings within the extended set A.

competition between the onset of $\Lambda$ and $\Sigma^{-}$depending on the $L$, with smaller values of $L$ favoring the $\Sigma^{-}$hyperon (see top figure of the right column).

As discussed in 13,14, a smaller slope $L$ implies a softer increase of the strangeness fraction with density. However, once the central density of these stars is larger, it is important to study their total hyperon content. This will be done by calculating for each star the total strangeness number

$$
N_{S}=4 \pi \int_{0}^{R} \frac{\rho_{s} r^{2}}{\sqrt{1-2 m(r) / r}} d r
$$

where $m(r)$ is the mass inside the radius $r$.

In Fig. 16 we plot the radius of a star with a mass $1.67 M_{\odot}$ similar to the mass of the pulsar PSR J1903+0327 $\left(1.67 \pm 0.02 M_{\odot}\right)[80$ as a function of its strangeness content. The largest strangeness fractions were obtained considering an attractive potential for the $\Sigma^{-}$meson. It is interesting to notice that two almost parallel straight lines are obtained: for $L=110 \mathrm{MeV}$ the slope is $-11.27 \pm 4 \%$ $\mathrm{km}$ and for $L=55 \mathrm{MeV}$ the slope is $-10.62 \pm 1 \% \mathrm{~km}$. The straight lines cross the vertical axis for a nucleonic star with no hyperons. The slope is almost independent of $L$.

Finally, in Fig. 17] we show some properties of maximum mass stars. Some general conclusions may be drawn with respect to the strangeness content: a) the maximum star mass changes with $L$, stars with an intermediate $L$ have the smallest masses and, generally, have the largest central densities (see panels a) and e)). There are two competing factors that define this behavior: on one hand a larger $L$ corresponds to a harder EOS because the symmetry energy increases faster with the density, on the other hand a larger $L$ favors larger strangeness fractions which softens the EOS. The first one gives rise to smaller central densities and larger radii, while the second one leads to the opposite; b) the strangeness content depends on the hyperon interaction, and, in particular, on the $\Xi$ potential in the present study. If $U_{\Xi}=+18 \mathrm{MeV}$ (triangles) the masses are larger and the strangeness fractions generally smaller; c) the inclusion of the strange mesons gives rise to more massive stars that may have larger strangeness contents. In this case the strangeness content is always smaller for a smaller slope $L$, and its maximum value is of the order 0.04-0.05 according to the hyperon interaction if $L=55 \mathrm{MeV}$. The upper limit can reach 0.07-0.08 if $L=110 \mathrm{MeV}$, Fig. 17d ). Larger fractions may be obtained if the $U_{\Sigma}$ is considered attractive.

\section{Conclusions}

We have studied the effect of the density dependence of the symmetry energy on several properties of neutron stars. In particular, we have discussed the properties of the crustcore transition, the pasta phase, and a possible existing competition between the effects of the symmetry energy and exotic degrees of freedom in the EOS.

The problems have been investigated within different nuclear matter approaches, namely the BHF one, several Skyrme forces, RMF models and a generalized liquid drop model.

First, we have analyzed the correlations of the slope parameter $L$ with the core-crust transition from homogeneous to clusterized matter in neutron stars, using a simplified definition of this transition, namely the crossing between the line of beta equilibrium and the thermodynamical spinodal. It was shown that the core-crust transition density and proton fraction appear clearly correlated with $L[7,8$. On the other hand no clear correlation was observed between $L$ and the transition pressure.

We have shown that the determination of the corecrust transition by the crossing between the dynamical spinodal and the $\beta$-equilibrium corresponds to a realistic approximation, with results very similar to the TF prediction. It takes place at a density lower than expected if the thermodynamical spinodal approach is applied, however, it was confirmed that the correlations obtained within the thermodynamical approach are still valid using the dynamical spinodal approach.

We have verified that the predictivity of the transition pressure is considerably improved in terms of selected pairs of coefficients. In particular, a strong correlation appears between the transition pressure and a combination of the symmetry energy slope and curvature parameters at the same reference density, $\rho=0.1 \mathrm{fm}^{-3}$. This correlation indicates that the relation between nuclear observables and the liquid drop model coefficients should be investigated at subsaturation densities.

In the second part, we have studied the inner-crust properties of neutron stars within a self-consistent ThomasFermi approach developed in Refs. [56, 57, for relativistic nuclear models, and the coexisting-phases method [10,56]. Several relativistic nuclear models have been used, with nonlinear meson terms and constant couplings, or with density-dependent coupling constants.

The properties of the models used are reflected in the cluster structure. It was seen that a small symmetry energy slope $L$ gives rise to larger cells, with a larger proton and neutron number, while the opposite occurs for a large 

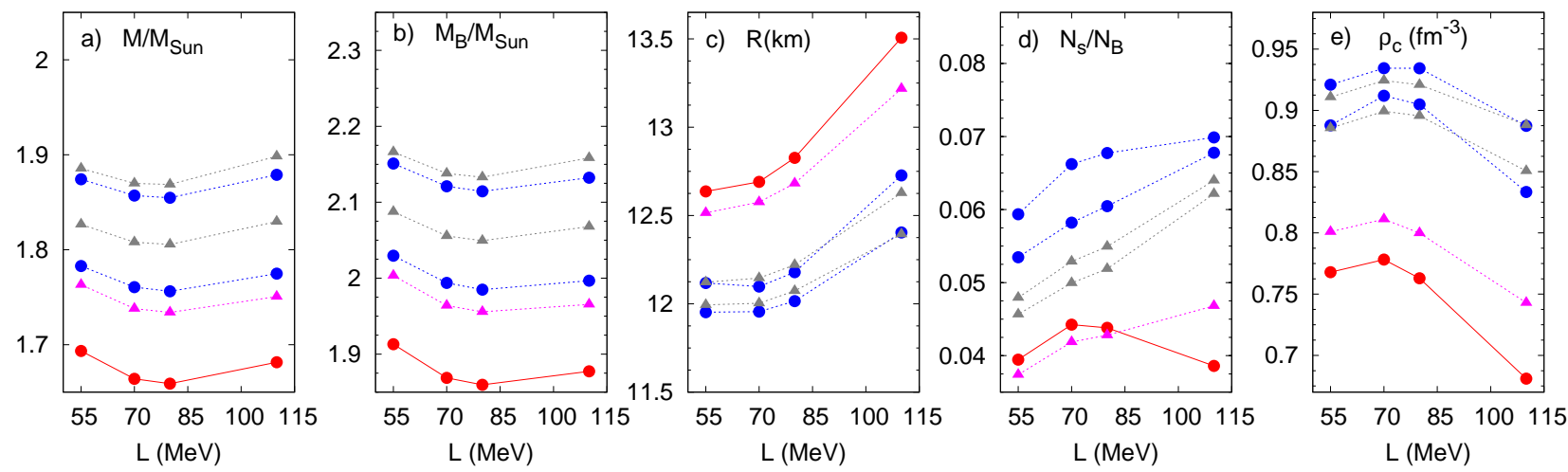

Fig. 17. (Color online) Properties of maximum mass stars obtained with $L=55,70,80$ and $110 \mathrm{MeV}$, for TM1 and extended set A: $U_{\Xi}=+18 \mathrm{MeV}$ (triangles) without (pink) and with (grey) $Y Y$ interaction; $U_{\Xi}=-18 \mathrm{MeV}$ (circles) without (red) and with (blue) $Y Y$ interaction. For the YY interaction, two choices are considered, as discussed in the text. For all the curves, $U_{\Lambda}=-28 \mathrm{MeV}$ and $U_{\Sigma}=30 \mathrm{MeV}$. The panels contain: a) gravitational mass, b) baryonic mass, c) star radius, d) strangeness content and e) central density.

L. In particular, it was shown that the NL3 model, with a very large symmetry energy and slope, and the IU-FSU one, with a quite small slope, have very different behaviors. NL3 does not present non-droplet pasta phases in the inner crust of $\beta$-equilibrium matter, and predicts the smallest proton and neutron numbers and Wigner-Seitz radii in almost all the inner-crust range of densities. On the contrary, IU-FSU predicts a quite low density for the onset of the non-droplet pasta phase, the largest crust-core transition density, and the largest clusters.

All models, except NL3, predict the existence of lasagnalike structures that may have an important contribution to the specific heat of the crust 12 .

The effect of the inner crust EOS on the neutron star profile was also analysed. It was verified that a smaller slope gives rise to a steeper crust density profile and a larger inner crust with respect to the total crust. It may also enhance the slab phase extension as observed in IUFSU.

Finally, in the last part we discussed the joint effect of strangeness and the symmetry energy on some properties of the neutron stars, such as the hyperon content, DU and radius.

It was shown that the smaller the slope $L$, the larger the onset density of the DU process. However, the DU onset also depends on the hyperon content and the hyperonmeson couplings. The DU may be hindered or favored according to a balance between the neutron and proton reductions if a neutral hyperon sets on first. Negatively charged hyperons favor the DU onset due to a decrease of the neutron fraction and an increase of the proton fraction.

It was also shown that, for a star with a fixed mass, the radius of the star decreases linearly with the increase of the total strangeness content. In particular, a $1 \mathrm{~km}$ decrease of the radius of a $1.67 M_{\odot}$ star may be explained if the slope of the symmetry energy decreases from 110 to $55 \mathrm{MeV}$ or the strangeness to baryon fraction increases from zero to 0.09 .
A softer symmetry energy corresponds to a slower increase of the hyperon fraction with density. However, the onset of strangeness depends on the charge of the hyperons. Negatively charged hyperons set on at smaller densities while neutral hyperons appear at larger densities for smaller values of the slope.

If a repulsive hyperon-hyperon interaction is considered, although a larger slope $L$ gives rise to a larger strangeness content, the extra repulsion between hyperons compensates the extra hyperon fraction and the effect of the symmetry energy is almost not seen on the central density of the maximum mass configuration.

We conclude that some star properties are affected in a similar way by the density dependence of the symmetry energy and the hyperon content of the star. To disentangle these two effects it is essential to have a good knowledge of the EOS at suprasaturation densities. There is still lack of information about the nucleonic EOS at suprasaturation densities as well as on the hyperon interactions in nuclear matter that may allow for an unambiguous answer to whether the mass of the pulsars J1614-2230 81] or J0348+0432 82 could rule out exotic degrees of freedom from the interior of compact stars.

The symmetry energy density dependence and its slope have been topics of intense investigation in the latest years. The work we have just presented in this paper is intrinsically related to other topics also discussed in this special volume. The search for constraints to the huge variety of equations of states used to describe neutron star matter involves astrophysical observations, heavy ion collision data, nuclear reactions 83 84, nuclear structure, bulk matter empirical values 39 and finite nuclei properties, as the neutron skin thickness 85, 86. On the other hand, the strangeness content of different equations of state, shown above to be related to the symmetry energy, has important consequences on both the liquid-gas phase transition and the transition at high densities 87. Hence, further investigation towards a better understanding of the symmetry energy density dependence is still required. 


\section{Acknowledgements:}

This work was carried out within the R\&DT projects PTDC/FIS/113292/2009 and CERN/FP/123608/2011, developed under the scope of a QREN initiative, UE/FEDER financing, through the COMPETE programme and by the NEW COMPSTAR, a COST initiative.

\section{References}

1. V. Baran, M. Colonna, V. Greco, and M. Di Toro, Phys. Rep. 410, 335 (2005).

2. L. W. Chen, C. M. Ko and B. A. Li, Phys. Rev. Lett. 94, 032701 (2005); Phys. Rev. C 72, 064309 (2005); B. A. Li, L. W. Chen, C. M. Ko, Phys. Rep. 464, 113 (2008).

3. A. W. Steiner, M. Prakash, J. Lattimer, and P. J. Ellis, Phys. Rep. 411, 325 (2005).

4. W. G. Lynch (unpublished), http://www.smith.edu/nusym11/program.php

5. B. A. $\quad \mathrm{Li}$ (unpublished), http://www.smith.edu/nusym11/program.php

6. I. Vidaña, C. Providência, A. Polls and A. Rios, Phys. Rev. C 80, 045806 (2009).

7. C. Ducoin, J. Margueron and C. Providência, Eur. Phys. Lett. 91, 32001 (2010).

8. C. Ducoin, J. Margueron, C. Providência and I. Vidaña, Phys. Rev. C 83, 45810 (2011).

9. S. S. Avancini, S. Chiacchiera, D. P. Menezes, and C. Providência Phys. Rev. C 82, 055807 (2010); Phys. Rev. C 85, 059904 (2012).

10. S. S. Avancini, C. C. Barros, Jr., L. Brito, S. Chiacchiera, D. P. Menezes, and C. Providência Phys. Rev. C 85, 035806 (2012).

11. F. Grill, C. Providência, and S. S. Avancini Phys. Rev. C 85, 055808 .

12. L. Di Gallo, M. Oertel, and M. Urban, Phys. Rev. C, 84, 045801 (2011).

13. R. Cavagnoli, C. Providência, D. P. Menezes Phys. Rev. C 84, 065810 (2011).

14. P. K. Panda, A. M. S. Santos, D. P. Menezes, C. Providência, Phys. Rev. C 85, 055802 (2012).

15. C. Providência, R. Cavagnoli, D. P. Menezes, P. K. Panda and A. Rabhi, J. Phys. Conf. Ser. 413, 012023 (2013).

16. C. Providência and A. Rabhi, arXiv:1212.5911 [nucl-th], to appear in Phys. Rev. C

17. I. Bombaci and U. Lombardo, Phys. Rev. C 44, 1892 (1991).

18. J. P. Jekeunne, A. Lejeunne and C. Mahaux, Phys. Rep. 25, 83 (1976).

19. R. B. Wiringa, V. G. J. Stoks and R. Schiavilla, Phys. Rev. C 51, 38 (1995).

20. B. A. Loiseau, Y. Nogami, and C. K. Ross, Nucl. Phys. A 165, 601 (1971); 176, 665(E) (1971); P. Grangé, M. Martzolff, Y. Nogami, D. W. L. Sprung and C. K. Ross, Phys. Lett. B 60, 237 (1976); M. Baldo and L. Ferreira, Phys. Rev. C 59, 682 (1999).

21. D. Vautherin and D. M. Brink, Phys. Rev. C 3, 626 (1972); P. Quentin and H. Flocard, Annu. Rev. Nucl. Part. Sci. 28, 523 (1978).

22. B. D. Serot and J. D. Walecka, Adv. Nucl. Phys. 16, 1 (1986); Int. J. Mod. Phys. E 6, 515 (1997).

23. J. R. Stone, J. C. Miller, R. Koncewicz, P. D. Stevenson, and M. R. Strayer, Phys. Rev. C 68, 034324 (2003).
24. E. Chabanat, Ph.D. thesis, Université Claude Bernard Lyon-1, 1995, Report No. LYCENT 9501 (unpublished).

25. E. Chabanat, P. Bonche, P. Haensel, J. Meyer, and R. Schaeffer, Nucl. Phys. A 627, 710 (1997); ibid, Nucl. Phys. A 635, 231 (1998).

26. P.-G. Reinhard, and H. Flocard, Nucl. Phys. A 584, 467 (1995).

27. W. Nazarewicz, J. Dobaczewski, T. R. Werner, J. A. Maruhn, P.-G. Reinhard, K. Rutz, C. R. Chinn, A. S. Umar, and M. R. Strayer, Phys. Rev. C 53, 740 (1996).

28. J. Friedrich, and P.-G. Reinhard, Phys. Rev. C 33, 335 (1986).

29. N. Van Giai, and H. Sagawa, Phys. Lett. B 106, 379 (1981).

30. L. Bennour, P. Bonche, J. Dobaczewski, and H. Flocard, Phys. Rev. C 40, 2834 (1989).

31. P.-G. Reinhard, D. J. Dean, W. Nazarewicz, J. Dobaczewski, J. A. Maruhn, and M. R. Strayer, Phys. Rev. C 60, 014316 (1999).

32. F. Tondeur, M. Brack, M. Farine, and J. M. Pearson, Nucl. Phys. A 420, 297 (1984).

33. M. Beiner, H. Flocard, N. Van Giai, and P. Quentin, Nucl. Phys. A 238, 29 (1975).

34. M. Rayet, M. Arnould, G. Paulus, and F. Tondeur, Astron. Astrophys. 116, 183 (1982).

35. L. G. Cao, U. Lombardo, C. W. Shen, and N. Van Giai, Phys. Rev. C 73, 014313 (2006).

36. S. Goriely, M. Samyn, and J. M. Pearson, Phys. Rev. C 75, 064312 (2007).

37. N. Chamel, S. Goriely, and J. M. Pearson, Nucl. Phys. A 812, 72 (2008).

38. S. Goriely, N. Chamel, and J. M. Pearson, Phys. Rev. Lett. 102, 152503 (2009).

39. M. Dutra, O. Lourenço, J. S. Sá Martins, A. Delfino, J.R. Stone and P.D. Stevenson, Phys. Rev. C 85, 035201 (2012).

40. J. Boguta and A. R. Bodmer, Nucl. Phys. A 292, 413 (1977).

41. S. Typel and H. H. Wolter, Nucl. Phys. A656, 331 (1999).

42. G. A. Lalazissis, J. König, and P. Ring, Phys. Rev. C 55, 540 (1997).

43. Y. Sugahara and H. Toki, Nucl. Phys. A 579, 557 (1994).

44. C. J. Horowitz and J. Piekarewicz, Phys. Rev. C 64062802 (2001); C. J. Horowitz and J. Piekarewicz, Phys. Rev. Lett. 86, 5647 (2001)

45. N. K. Glendenning and S. A. Moszkowski, Phys. Rev Lett. 67, 2414 (1991).

46. B. G. Todd-Rutel and J. Piekarewicz, Phys. Rev. Lett. 95, 122501 (2005).

47. F. J. Fattoyev, C. J. Horowitz, J. Piekarewicz, and G. Shen, Phys. Rev. C 82, 055803 (2010).

48. G. A. Lalazissis, T. Niksić, D. Vretenar, and P. Ring, Phys. Rev. C 71, 024312 (2005).

49. X. Roca-Maza, X. Viñas, M. Centelles, P. Ring, and P. Schuck, Phys. Rev. C 84, 054309 (2011).

50. B. Link, R.I. Epstein, J.M. Lattimer, Phys. Rev. Lett. 83 (1999) 3362.

51. C. Providência, L. Brito, S. S. Avancini, D. P. Menezes, and Ph. Chomaz Phys. Rev. C 73, 025805; L. Brito, C. Providência, A. M. Santos, S. S. Avancini, D. P. Menezes, and Ph. Chomaz, Phys. Rev. C 74, 045801 (2006); H. Pais, A. Santos, L. Brito, and C. Providência Phys. Rev. C 82, 025801 (2010). 
52. S. S. Avancini, L. Brito, Ph. Chomaz, D. P. Menezes, C. Providência, Phys. Rev. C 74, 024317, (2006).

53. C. Ducoin, C. Providência, A. M. Santos, L. Brito and Ph. Chomaz, Phys. Rev. C 78, 055801 (2008).

54. I. Vidaña and A. Polls, Phys. Lett. B 666, 232 (2008).

55. J. Xu, L. W. Chen, B. A. Li and H. R. Ma, Astrophys. J. 697, 1549 (2009).

56. S. S. Avancini, D. P. Menezes, M. D. Alloy, J. R. Marinelli, M. M. W. Moraes, and C. Providência, Phys. Rev. C 78, 015802, (2008).

57. S.S. Avancini, L. P. Brito, J.R. Marinelli, D.P. Menezes, M.M.W. Moraes, C. Providência and A. M. Santos, Phys. Rev. C 79, 035804 (2009).

58. C. J. Pethick, D. G. Ravenhall, C. P. Lorentz, Nucl. Phys. A 584, 675 (1995).

59. Ch. C. Moustakidis, T. Nitsic̀, D. Vretenar and P. Ring, Phys. Rev. C 81, 065803 (2010)

60. W. G. Newton, M. Gearheart and Bao-An Li, ApJ Supp. 204, 9 (2013)

61. D. G. Ravenhall, C.J. Pethick and J.R. Wilson, Phys. Rev. Lett. 50, 2066 (1983).

62. C.J. Horowitz, M.A. Pérez-Garcia and J. Piekarewicz, Phys. Rev. C 69, 045804 (2004); C.J. Horowitz, M.A. Pérez-Garcia, D.K. Berry and J. Piekarewicz, Phys. Rev. C 72, 035801 (2005).

63. T. Maruyama, T. Tatsumi, D.N. Voskresensky, T. Tanigawa and S. Chiba, Phys. Rev. C 72, 015802 (2005).

64. G. Watanabe, T. Maruyama, K. Sato, K. Yasuoka, and T. Ebisuzaki, Phys. Rev. Lett. 94, 031101 (2005).

65. H. Sonoda, G. Watanabe, K. Sato, K. Yasuoka, and T. Ebisuzaki, Phys. Rev. C 77, 035806 (2008); Phys. Rev. C 81, 049902 (2010).

66. J. $\mathrm{Xu}, \mathrm{L} . \mathrm{W}$. Chen, B.A. $\mathrm{Li}$ and H.R. Ma, arXiv:0807.4477 1 [nucl-th].

67. K. Oyamatsu and K. Iida, Phys. Rev. C 75, 015801, (2007).

68. B. A. Brown, Phys. Rev. Lett. 85, 5296 (2000); S. Typel and B. A. Brown, Phys. Rev. C 64, 027302 (2001).

69. M. Hempel and J. Schaffner-Bielich, Nucl. Phys. A 837, 210 (2010).

70. Ad. R. Raduta and F. Gulminelli, Phys. Rev. C 82, 065801 (2010).

71. F. Grill, S. S. Avancini, S. Chiacchiera, C. Providência, I. Vidaña, in preparation.

72. G. Baym, C. Pethick, and P. Sutherland, Astrophys. J. 134, 683 (1971)

73. M. Gearheart, W. G. Newton, J. Hooker, and Bao-An Li, MNRAS 418, 2343 (2011)

74. P. Danielewicz, R. Lacey, and W. G. Lynch, Science 298, 1592 (2002).

75. Chiapparini M et al, Nucl. Phys. A 826, 178 (2009).

76. J. Schaffner-Bielich and A. Gal, Phys. Rev. C 62, 034311 (2000); J. Schaffner-Bielich, M. Hanauske, H. Stocker and W. Greiner, Phys. Rev. Lett. 89, 171101, (2002); E. Friedman and A. Gal, Phys. Rep. 452, 89 (2007).

77. A. Gal, Prog. Theor. Phys. Suppl. 186, 270 (2010) and references therein

78. A. Gal and D. Millener, Phys. Lett. B 701, 342 (2011).

79. R. Cavagnoli and D. P. Menezes, J. Phys. G 35, 115202 (2008).

80. P. C. C. Freire et al., MNRAS 412, 2763 (2011)

81. Paul Demorest, Tim Pennucci, Scott Ransom, Mallory Roberts, Jason Hessels, Nature 467, 1081 (2010).
82. Antoniadis, J.; Freire, P. C. C.; Wex, N.; Tauris, T. M.; Lynch, R. S.; Van Kerkwijk, M. H.; Kramer, M.; Bassa, C. et al. (2013) Science 340 (6131): 1233232.

83. J.M. Lattimer and Y. Lim, arXiv:1203.4286v1.

84. M.B. Tsang et. al., Phys. Rev. C 86, 015803 (2012).

85. F. J. Fattoyev and J. Piekarewicz, Phys. Rev. C 86, 015802 (2012).

86. X. Roca-Maza, M. Centelles, X. Viñas and M. Warda, Phys. Rev. Lett. 106, 252501 (2011).

87. F. Gulminelli, Ad. R. Raduta, M.Oertel and J. Margueron, Phys. Rev. C 87, 055809 (2013). 
